\title{
CIRCULAR PLATES OF CONSTANT OR VARIABLE THICKNESS*
}

BY

\author{
CARI A. GARABEDIAN
}

CONTENTs

Part I. The theory Page

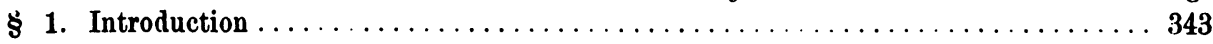

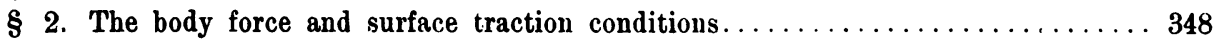

$\$ 3$. Determination of formulas of displacement that satisfy the body force conditions 351

$\$$ 4. Four systems of differential equations obtained from the surface traction conditions 358

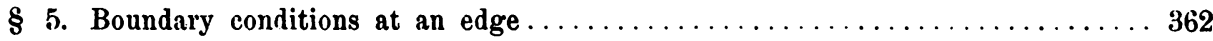

$\$$ 6. Analysis of the formulas of displacement by the aid of certain transformations 368

Part II. Applications

$\$ 7$. Incomplete circular plate under internal and external radial pressure ....... 373

$\$$ 8. Incomplete circular plate of variable thickness under internal and external radial

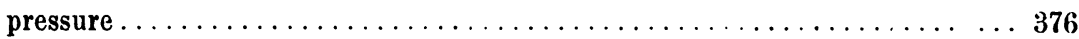

§ 9. Complete circular plate loaded at its center and clamped at the elge...... 379

$\$ 10$. Complete circular plate loaded at its center and supported at the edge ..... 381

§11. Complete circular plate under uniform pressure and clamped at the edge .... 384

\$12. Complete circular plate under uniform pressure and supported at the edge ... 386

\$13. Plates of constant thickness under pressure of type such that the series for the

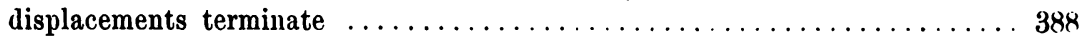

\$14. Incomplete circular plate under uniforn pressure with one edge clamped and

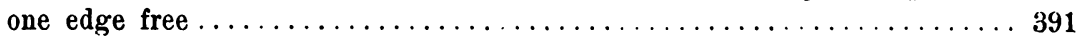

\$15. Incomplete circular plate of variable thickness under uniform pressure with one

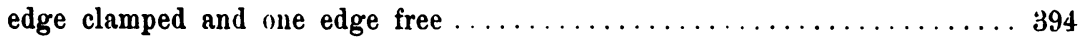

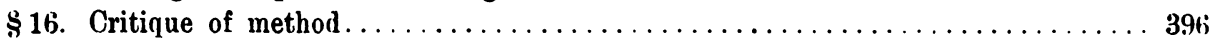

\section{PART I. THE THEORY}

1. Introduction. In a recent notet on Circular plates of rariable triclines:; Professor Birkhoff points the way to a new method in the theory of elasticity. Cauchył treated the case of variable thickness, but like Poisson§ he assumed the displacements developable in ascending positive integral powers of the

* Presented to the American Mathematical Society, February 24, 1923. See also ('omptes Rendus, vol. 177, No. 20, Nov. 12, 1925.

† Philosophical Magazine, ser. 6, No. 257, May, 1922.

† Exercices de Mathématique, vol. 3 (1828), pp. 277-284.

$\$$ Memoir of 1828. Cf. also Todhunter and Pearson's History. vol. 1. 
distance from the middle-surface. Saint-Venant* and others were quick to observe that this assumption is quite untenable, and it appears that subsequent investigations in the direction taken by Poisson and Cauchy have as yet failed to reveal a suitable type of series development. $t$ With reference to the question here at issue, it may be said that the object of the present paper is to present a powerful and direct method of series based upon the introduction of the "natural" parameter $t$ employed by Birkhoff. The vital contribution of Birkhoff's note seems to be the notion of expansions in power series in $t$.

A first exposition is simplified by assuming the conditions such that the displacements take place in planes through an axis and are the same in all such planes. The advantage of this assumption is that the differential equations to be integrated are essentially total. Cylindrical coördinates are adopted, with the axis of $z$ along the axis of the plate; and although the thickness is variable, it is assumed that there is symmetry in the "middle plane" $z=0$. The plate is taken to be homogeneous and isotropic, only slightly bent, $\$$ and in general thin.

Let the equations of the upper and lower bases of the plate be respectively

$$
\begin{aligned}
& z=a(r), \\
& z=-a(r) .
\end{aligned}
$$

The method of series begins with the introduction of a parameter $t$ by means of the relation

$$
z=5 t \text {. }
$$

At the same time we write

$$
a(r)=\alpha(r) t,
$$

* De Clebsch, Théorie de l'Elasticité des C'orps Solides, 1883, annotated by Saint-Venant. Cf. also Love, $A$ Treatise on the Mathematical Theory of Elasticity, third edition, 1920, p. 27.

$\dagger$ As this paper nears completion, I find a note by E. and M. Cosserat (Comptes Rendus, vol. 146, (1908), p. 169) which seems to be the most recent contribution to the subject in hand. Although this note recognizes the fact that the displacements are functions not only of the coördinates but also of certain geometric parameters, the writers deal only briefly with the question of series developments. They investigate the character of the displacements in the case of classic plate and rod problems, but in their method of attack there is no suggestion of series developments of the type on which we base the systematic treatment of the present paper. Messrs. Cosserat make no mention of plates of variable thickness or of rods of variable cross section.

$\ddagger$ We shall exclude from the present paper any discussion of plates not symmetrical in $z=0$.

$\$$ Love, p. 462. 
so that the equations of the bases may be written in the form

$$
\begin{aligned}
& z=\alpha t \\
& z=-\alpha t
\end{aligned}
$$

or, when convenient, in the form

$$
\zeta=\alpha,
$$

$$
\zeta=-\alpha
$$

Our fundamental assumption is that all quantities involved which are functions of $r$ and $z$ can be expanded in ascending powers of the parameter $t$; in particular, that

$$
\begin{aligned}
& U(r, z)=U_{0}(r, \zeta)+U_{1}(r, \zeta) t+J_{2}(r, \zeta) t^{2}+\cdots \\
& w(r, z)=w_{0}(r, \zeta)+w_{1}(r, \zeta) t+v_{2}(r, \zeta) t^{2}+\cdots
\end{aligned}
$$

where $U$ and $w$ denote the radial and axial displacements respectively.

Let us write $a(r)=h f(r), \alpha(r)=\eta f(r)$, where $h=\eta t$ is a constant multiplier. If $f(r) \equiv 1$, we have the special case of constant thickness: $a=h, \alpha=\eta$. With this notation, it turns out that the coefficient of $t^{n}$ in $(6 a)$ or $(6 b)$ is a homogeneous polynomialt in $\zeta$ and $\eta$ of degree $n$; that is, when the return is made to the original variable, the terms are homogeneous polynomials in $z$ and $h$ ordered according to degree. The coefficients are functions of $r$ and the fixed radii of the plate, of $f$ and its derivatives, and of the elastic constants and applied tractions. The manner in which these arguments enter is of no immediate concern, but it should be noted that the requirement of homogeneity in $\zeta$ and $\eta$ places a very definite restriction upon the manner of occurrence of $f$ and its derivatives. We shall find occasion in the sequel to make further comments with reference to the structure of our formulas.

The $t$ plays a rôle analogous to that of the parameter in the definition of homogeneous function. Although a physical interpretation of transformation (2) is not immediate, there is suggested the picture of a "plate of reference" whose bases have the equations (5) and whose thickness is controlled by means of the parameter $t$. Or we may think of (5) as fixed, in which case the

$\dagger$. See the examples of Part II. 
plate (4) has its thickness determined by $t$, and upon proper choice of $t$ becomes the given plate.

The assumption of Cauchy and Poisson of developability in powers of $z$ alone involves suppression of terms in the geometric parameter $h$. The introduction of the parameter $t$ furnishes a method that incurs no loss of terms; and when the parameter $t$ has served its purpose, it may be suppressed by setting $t=1$ and replacing the Greek letters $\zeta$ and $\eta$ by $z$ and $h$, respectively.

We shall find it convenient to hold in abeyance the energy integral and calculus of variations method employed by Birkhoff. and proceed directly from the fundamental equations of elasticity. We take the point of view of prescribed pressures on the faces of the plate, and compute the displacements. This type of problem will require that the coefficients in the formulas of displacement be so determined that

(i) the body forces have the required values,

(ii) the surface tractions on the bases are as prescribed,

(iii) the boundary conditions at the edges are satisfied.

Our procedure is to be entirely in accord with the accepted elastic theory, as set forth, for example, by Love. Requirements (i), (ii), and (iii) are characterized by the usual equations, except that from our point of view they become identities in $t$. That which is new is $(a)$ the method of proceeding from these equations to the actual solution of a problem, and $(b)$ the applicability of the method to plates of variable thickness.

As a matter of notation, it is convenient to introduce an operator defined as followst:

$$
\begin{aligned}
A^{*} & =\frac{1}{r} \frac{\partial}{\partial r}(r \cdot A), \\
& =A^{\prime}+\frac{A}{r} .
\end{aligned}
$$

† In the literature (Love, p. 202) one finds the Laplacian operators

$$
\begin{aligned}
& \nabla_{i}^{2}=\frac{\partial^{2}}{\partial x^{2}}+\frac{\partial^{2}}{\partial y^{2}} \\
& \nabla_{i}^{4}=\frac{\partial^{4}}{\partial x^{4}}+\frac{\partial^{4}}{\partial y^{4}}+2 \frac{\partial^{4}}{\partial x^{2} \partial y^{2}}
\end{aligned}
$$

when $A$ is a function of $r$ only, where $r^{2}=x^{2}+y^{2}$, it may be verified that

$$
\begin{aligned}
& \nabla_{1}^{2} A=A^{\prime *}, \\
& \nabla_{1}^{*} A=A^{\prime * * *}
\end{aligned}
$$


Here the subject of operation is in general a function of both $r$ and $\zeta$, and the accent denotes differentiation with regard to $r$. The functions that involve both $r$ and $\zeta$ turn out to be polynomials in $\zeta$, and consequently the operator and symbol of differentiation are attached ultimately to functions of $r$ alone. Since the operator and the sign of differentiation occur frequently in alternation, we write $\left(\left[\left(A^{*}\right)^{\prime}\right]^{*}\right)^{\prime}$ in the more compact form: $A^{* \prime * \prime}$. For reference we note also the formulas

$$
\begin{aligned}
(A B)^{*} & =A^{*} B+A B^{\prime} \\
& =A B^{*}+A^{\prime} B=(B A)^{*}
\end{aligned}
$$

In integrating an equation of the type

$$
A^{* * *}=f(r)
$$

we make use of the form of definition $(7 a)$, and writet

$$
\frac{1}{r} \frac{d}{d r}\left[r \frac{d}{d r}\left\{\frac{1}{r} \frac{d}{d v}\left(r \frac{d A}{d r}\right)\right\}\right]=f(r),
$$

from which $A$ is readily found by a succession of quadratures. For example, the solution of

$$
A^{*} \cdots B
$$

is

$$
A=\frac{1}{r} \int(r B) d r+\frac{C}{r},
$$

$C$ being a constant of integration.

Just as we speak of differentiating or integrating a function, it is useful to say that we "star" a function by multiplying by the independent variable, differentiating, and dividing by the independent variable; likewise, that we "anti-star" a function by multiplying by the independent variable, integrating, and dividing by the independent variable.

The object of the present paper is to develop enough of the formal machinery involved in our method to enable us to treat some important and interesting applications to plates meeting the requirements of symmetry already outlined. We shall consider first some cases of uniform thickness, in order both to check

† Clebsch, p. 735. 
with the classical results and to gain familiarity with the details of the method from a formal viewpoint. Secondly, we shall give concrete examples sufficiently illustrative of the applications to plates of variable thickness.

What new light this program may shed upon the theory of circular plates of constant or variable thickness remains to be seen. We proceed at once to a systematic treatment along the lines indicated.

2 . The body force and surface traction conditions. Using the star notation, the body force conditionst are given by

$$
\begin{aligned}
& (\lambda+2 \mu) U^{*^{\prime}}+\frac{\lambda+\mu}{t} \frac{\partial w^{\prime}}{\partial \zeta}+\frac{\mu}{t^{2}} \frac{\partial^{2} U}{\partial \zeta^{2}} \overline{\bar{t}}-\varrho F_{r}, \\
& \frac{\lambda+2 \mu}{t^{2}} \frac{\partial^{2} w}{\partial \zeta^{2}}+\frac{\lambda+\mu}{t} \frac{\partial U^{*}}{\partial \zeta}+\mu w^{\prime *} \overline{\bar{t}}-\varrho F_{\boldsymbol{z}} .
\end{aligned}
$$

Here the displacements $U$ and $w$ are given by (6). To simplify matters, we shall later take the body forces to be nil, but in general $F_{r}$ and $F_{z}$ are assumed developable in suitable power series in $t$ of type (6). The relations (12) are identities in $t$, and the fact that coefficients of like powers of $t$ are the same gives us our first hold on the coefficients that enter in (6).

To gain compactness in later formulas, it is desirable to introduce at once the elastic constants $E$ and $\sigma$, denoting Young's modulus and Poisson's ratio respectively. $\neq$ For reference we note that

$$
\begin{array}{ll}
E=\frac{\mu(3 \lambda+2 \mu)}{\lambda+\mu}, & \sigma=\frac{\lambda}{2(\lambda+\mu)} \\
\lambda=\frac{E \sigma}{(1+\sigma)(1-2 \sigma)}, & \mu=\frac{E}{2(1+\sigma)} .
\end{array}
$$

The identities (12) may now be written in the form§

(14a) $2(1-\sigma) U^{*^{\prime}}+\frac{1}{t} \frac{\partial w^{\prime}}{\partial \zeta}+\frac{1-2 \sigma}{t^{2}} \frac{\partial^{2} U}{\partial \zeta^{2}} \overline{\overline{\bar{t}}}-\frac{2(1+\sigma)(1-2 \sigma) \varrho F_{r}}{E}$,
$(14 b) \frac{2(1-\sigma)}{t^{2}} \frac{\partial^{2} w}{\partial \zeta^{2}}+\frac{1}{t} \frac{\partial U^{*}}{\partial \zeta}+(1-2 \sigma) w^{*} \overline{\overline{\bar{t}}}-\frac{2(1+\sigma)(1-2 \sigma) \varrho F_{z}}{E}$.

† Love, p. 141.

$\ddagger$ Love, p. 101, § 69 .

$\S$ The equations (14) and (21) are divided by a constant factor as an aid to simplification of later formulas. 
To derive the conditions for tractions applied to the faces of the plate, consider a tangent plane at an arbitrary point $\left(r_{0}, \theta_{0}, z_{0}\right)$ of the upper surface, $z=a=t \alpha$, and denote by $\nu$ the direction of the normalt taken positively upwards. We describe the traction across the tangent plane to the surface $r=r_{0}$ by means of its vector components $(\widehat{r r}, \widehat{\theta r}, \widehat{z r})$, with a similar notation for the tractions across the planes $\theta=\theta_{0}$ and $z=z_{0}$. The first letter shows the direction of the component traction, and the second letter indicates the plane across which it acts. The sense $e_{+}^{+}$is such that $\widehat{r r}$ is positive when it is a tension, negative when it is a pressure. When the components of the traction across the tangent plane are expressed in terms of the stress-components across the planes normal to $r, \theta, z$, we have

$$
\begin{aligned}
& \widehat{r \nu}=\widehat{r r} \cos (r, v)+\widehat{r \theta} \cos (\theta, \nu)+\widehat{r z} \cos (z, \nu), \\
& \widehat{\theta \nu}=\widehat{\theta r} \cos (r, \nu)+\widehat{\theta \theta} \cos (\theta, \nu)+\widehat{\theta z} \cos (z, \nu), \\
& \widehat{z \nu}=\widehat{z r} \cos (r, \nu)+\widehat{z \theta} \cos (\theta, \nu)+\widehat{z z} \cos (z, \nu) .
\end{aligned}
$$

In cylindrical coördinates, the stress-components are given in terms of the strain-components and the displacements $U$ and $w$ as follows:\&

$$
\begin{aligned}
& \widehat{r r}=\lambda \Delta+2 \mu e_{r r}=\lambda \Delta+2 \mu U^{\prime}, \\
& \widehat{\theta \theta}=\lambda \Delta+2 \mu e_{\theta \theta}=\lambda \Delta+2 \mu \frac{U}{r}, \\
& \widehat{z z}=\lambda \Delta+2 \mu e_{x z}=\lambda \Delta+2 \mu \frac{1}{t} \frac{\partial w}{\partial \zeta}, \\
& \widehat{\theta z}=\widehat{z \theta}=\mu e_{\theta z}=0, \\
& \widehat{z r}=\widehat{r z}=\mu e_{z r}=\mu\left(\frac{1}{t} \frac{\partial U}{\partial \zeta}+w^{\prime}\right), \\
& \widehat{r \theta}=\widehat{\theta r}=\mu e_{r \theta}=0,
\end{aligned}
$$

$\dagger$ Love, p. 76.

$\ddagger$ Love, p. 76. Cf. Love, p. 461 , and also $\S 5$ of this paper.

\& Love, p. 101, 141. 
where

$$
\Delta=U^{*}+\frac{1}{t} \frac{\partial w}{\partial \zeta}
$$

and $U$ and $w$ are given by (6).

Since the slope of the tangent to $z=t \alpha$ is $t \alpha^{\prime}$, the desired direction cosines in (15) are

(17) $\cos (v, v)=\frac{-t \alpha^{\prime}}{\sqrt{1+t^{2} \alpha^{\prime 2}}}, \quad \cos (\theta, \nu)=0, \quad \cos (z, \nu)=\frac{1}{\sqrt{1+t^{2} \alpha^{\prime 2}}}$.

Let the applied tractions normal to the upper and lower bases be $\mathbf{S}^{\nu}$ and $\mathrm{S}_{\boldsymbol{v}}$ respectively, where

$$
\begin{aligned}
& \mathrm{S}^{v}=\mathrm{S}^{0}+\mathrm{S}^{1} t+\mathrm{S}^{2} t^{2}+\cdots, \\
& \mathrm{S}_{\nu}=\mathrm{S}_{0}+\mathrm{S}_{1} t+\mathrm{S}_{2} t^{2}+\cdots,
\end{aligned}
$$

the coefficients in these developments being functions of $r$ only. By virtue of (17) the radial and axial components may be written

$$
\begin{array}{ll}
\mathrm{R}^{\nu}=\frac{-t \alpha^{\prime} \mathrm{S}^{\nu}}{\sqrt{1+t^{2} \alpha^{\prime 2}}}, & \mathrm{Z}^{\nu}-\frac{\mathrm{S}^{\nu}}{\sqrt{1+t^{2} \alpha^{\prime 2}}} \\
\mathrm{R}_{\nu}=\frac{t \alpha^{\prime} \mathrm{S}_{\nu}}{\sqrt{1+t^{2} \alpha^{\prime 2}}}, \quad Z_{\nu}=\frac{\mathrm{S}_{\nu}}{\sqrt{1+t^{2} \alpha^{\prime 2}}} .
\end{array}
$$

If the plate is of constant thickness, $\alpha^{\prime}=0$ and $\mathrm{R}^{\nu} \equiv \mathrm{R}_{\nu} \equiv 0, \mathrm{Z}^{\nu} \equiv \mathrm{S}^{\nu}$, $\mathrm{Z}_{\nu} \equiv \mathrm{S}_{\nu}$.

We thus find for the surface tractions on the bases the identities

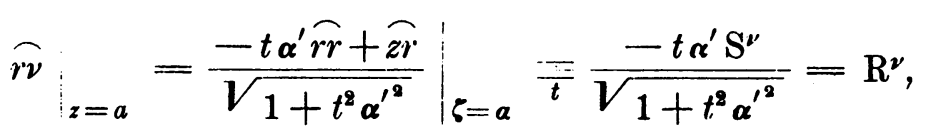

$$
\left.\widehat{r v}\right|_{z=-a}=\left.\frac{t \alpha^{\prime} \widehat{r r}+\overparen{z r}}{\sqrt{1+t^{2} \alpha^{\prime 2}}}\right|_{\zeta=-a} \bar{\equiv} \frac{t \alpha^{\prime} \mathrm{S}_{\nu}}{\sqrt{1+t^{2} \alpha^{\prime 2}}}=\mathrm{R}_{\nu}
$$

$$
\left.\widehat{z \nu}\right|_{z=a}=\left.\frac{-t \alpha^{\prime} \widehat{z r}+\overparen{z z}}{\sqrt{1+t^{2} \alpha^{\prime 2}}}\right|_{\zeta=a} \bar{\equiv} \frac{S^{\prime \prime}}{\sqrt{1+t^{2} \alpha^{\prime 2}}}=Z^{\nu}
$$

$$
\left.\widehat{z v}\right|_{z=-a}=\left.\frac{t \alpha^{\prime} \widehat{z v}+\widehat{z z}}{\sqrt{1+t^{2} \alpha^{\prime 2}}}\right|_{\zeta=-a} \frac{\mathrm{S}_{\nu}}{\sqrt{1+t^{2} \alpha^{\prime^{2}}}}=\mathrm{Z}_{\nu}
$$


Recall that the sense is such that the applied tractions are tensions when positive, pressures when negative.

Using formulas (16) and the elastic constants $E$ and $\sigma$, we have as final forms for the surface traction conditions the following:

$(21 a)$

$$
-2 \alpha^{\prime}\left\{\sigma\left(U^{*} t+\frac{\partial w}{\partial \zeta}\right)+(1-2 \sigma) U^{\prime} t\right\}
$$

$$
\begin{gathered}
+\left.(1-2 \sigma)\left\{\frac{1}{t} \frac{\partial U}{\partial \zeta}+w^{\prime}\right\}\right|_{\zeta=\alpha} \equiv \frac{-2(1+\sigma)(1-2 \sigma) t \alpha^{\prime} S^{\nu}}{E} \\
2 \alpha^{\prime}\left\{\sigma\left(U^{*} t+\frac{\partial w}{\partial \zeta}\right)+(1-2 \sigma) U^{\prime} t\right\}
\end{gathered}
$$

$$
\begin{aligned}
& +\left.(1-2 \sigma)\left\{\frac{1}{t} \frac{\partial U}{\partial \zeta}+w^{\prime}\right\}\right|_{\zeta=-\alpha} \overline{\overline{\bar{t}}} \frac{2(1+\sigma)(1-2 \sigma) t \alpha^{\prime}}{E} \\
& -\alpha^{\prime}(1-2 \sigma)\left\{\frac{\partial U}{\partial \zeta}+w^{\prime} t\right\}+2 \sigma U^{*}+\left.2(1-\sigma) \frac{1}{t} \frac{\partial w}{\partial \zeta}\right|_{\zeta=a} \\
& \overline{\overline{\bar{t}}} \frac{2(1+\sigma)(1-2 \sigma) \mathrm{S}^{\nu}}{E} \\
& \alpha^{\prime}(1-2 \sigma)\left\{\frac{\partial U}{\partial \zeta}+w^{\prime} t\right\}+2 \sigma U^{*}+\left.2(1-\sigma) \frac{1}{t} \frac{\partial w}{\partial \zeta}\right|_{\zeta=-\alpha} \\
& \overline{\bar{t}} \frac{2(1+\sigma)(1-2 \sigma) \mathrm{S}_{\nu}}{E} \text {. }
\end{aligned}
$$

3. Determination of formulas of displacement that satisfy the body force conditions. The body force conditions (14) will determine the coefficients in the formulas of displacement up to arbitrary functions of $r$. Next the traction conditions (21) come into play to furnish differential equations for the determination of these functions of $r$; and finally the constants of integration are fixed by means of the boundary conditions at the edges.

To handle expeditiously the program just outlined, it is desirable to introduce a notation. Let the equations obtained from $(14 a)$ and $(14 b)$ by equating coefficients of $t^{n}$ be denoted by $\mathrm{F}_{r}^{(n)}=0, \mathrm{~F}_{z}^{(n)}=0$, respectively. Thus the body force conditions yield the equations

$$
\mathrm{F}_{r}^{(n)}=0, \mathrm{~F}_{z}^{(n)}=0 \quad(n=-2,-1,0,1,2, \ldots)
$$


By an obvious extension of this notation, the traction conditions (21a), (21b), $(21 c),(21 d)$ may be said to yield respectively the equations

$$
\mathrm{R}^{(n)}=0, \mathrm{R}_{(n)}=0, \mathrm{Z}^{(n)}=0, \mathrm{Z}_{(n)}=0 \quad(n=-1,0,1,2, \ldots)
$$

We are now in a position to proceed to the determination of the coefficients in (6). The present paragraph is devoted to finding formulas of displacement that satisfy the identities (14) under the assumption that the body forces $F_{r}, F_{z}$ vanish identically.

From the equations $\mathrm{F}_{r}^{(-2)}=0, \mathrm{~F}_{z}^{(-2)}=0$ we find

$$
\frac{\partial^{2} U_{0}}{\partial \zeta^{8}}=0, \quad \frac{\partial^{2} w_{0}}{\partial \zeta^{2}}=0
$$

Integrating, we have

$$
\begin{aligned}
& U_{0}=U_{0 b}+U_{0 a} \zeta, \\
& w_{0}=w_{0 b}+w_{0 a} \zeta,
\end{aligned}
$$

where $U_{0 a}, U_{0 b}, w_{0 a}, w_{0 b}$ are functions of $r$ only, and are subsequently to be determined by means of the surface traction conditions.

In general, the equations $\mathrm{F}_{r}^{(n-2)}=0, \mathrm{~F}_{z}^{(n-2)}=0$ determine $U_{n}, w_{n}$ as polynomials in $\zeta$, with coefficients that are functions of the $U_{a}$ 's, $U_{b}$ 's, $u_{a}$ 's and $w_{b}$ 's. Integration will introduce two new arbitrary functions of $r$, and the coefficients of all powers of $\zeta$ higher than the first will be known in terms of functions of $r$ previously introduced; for example:

$$
\begin{aligned}
& U_{n}=U_{n b}+U_{n a} \zeta+(\text { terms of higher order in } \zeta), \\
& w_{n}=w_{n b}+w_{n a} \zeta+(\text { terms of higher order in } \zeta) .
\end{aligned}
$$

Finally, we note that the computation of $U_{n}$ will require that $w_{n-1}$ and $U_{n-2}$ should already have been computed, and that the computation of $w_{n}$ will require the previous computation of $U_{n-1}$ and $w_{n-2}$.

Before proceeding to (22), $n=-1$, an important simplification of (25) results if we first introduce the traction conditions (23), $n=-1$. These relations are found to be 


$$
\begin{aligned}
& \mathrm{R}^{(-1)}=\left.\frac{\partial U_{0}}{\partial \zeta}\right|_{\zeta=a}=0, \quad \mathrm{R}_{(-1)}=\left.\frac{\partial U_{0}}{\partial \zeta}\right|_{\zeta=-\alpha}=0 \\
& \mathrm{Z}^{(-1)}=\left.\frac{\partial w_{0}}{\partial \zeta}\right|_{\zeta=a}=0, \quad \mathrm{Z}_{(-1)}=\left.\frac{\partial w_{0}}{\partial \zeta}\right|_{\zeta=-\alpha}=0,
\end{aligned}
$$

and they require that $U_{0 a} \equiv w_{0 a} \equiv 0$; in other words, the leading terms in the formulas of displacement are independent of $\zeta$.

From $\mathrm{F}_{r}^{(-1)}=0, \mathrm{~F}_{z}^{(-1)}=0$, we have

$$
\begin{aligned}
& \frac{\partial^{2} U_{1}}{\partial \zeta^{2}}=-\frac{1}{1-2 \sigma} \frac{\partial w_{0}^{\prime}}{\partial \zeta} \\
& \frac{\partial^{2} w_{1}}{\partial \zeta^{2}}=-\frac{1}{2(1-\sigma)} \frac{\partial U_{0}^{*}}{\partial \zeta}
\end{aligned}
$$

but the right-hand members vanish, since $U_{0}=U_{0 b}$ and $w_{0}=w_{0 b}$ are functions of $r$ only, and we find

$$
\begin{aligned}
& U_{1}=U_{1 b}+U_{1 a} \zeta, \\
& w_{1}=w_{1 b}+w_{1 a} \zeta .
\end{aligned}
$$

Before proceeding to the computation of $U_{2}$ and $w_{2}$ it will simplify matters to consider the traction conditions (23) for $n=0$. To deal conveniently with these and subsequent traction equations, we introduce a further special notation. Add equations (21a) and (21b), and denote by $\mathrm{R}^{n}=0$ (without parentheses enclosing the superscript) the equation obtained by equating coefficients of $t^{n}$ in the resulting equation; similarly, let $\mathrm{R}_{n}=0$ denote the equation obtained by equating coefficients of $t^{n}$ in the equation which results when $(21 b)$ is subtracted from $(21 a)$. Finalıy, let $Z^{n}=0$ and $Z_{n}=0$ denote analogous equations derived from the sum and difference, respectively, of $(21 c)$ and $(21 d)$. The surface traction differential equations (23) become

$$
\mathrm{R}^{n}=0, \quad \mathrm{R}_{n}=0, \quad \mathrm{Z}^{n}=0, \quad \mathrm{Z}_{n}=0 \quad(n=-1,0,1,2, \cdots) .
$$

We have already disposed of the case $n=-1$; and since $R^{(0)}=0, R_{(0)}=0$ require that

$$
\frac{\partial U_{1}}{\partial \zeta}+\left.w_{0}^{\prime}\right|_{\zeta= \pm \alpha}=U_{1 a}+v_{0 b}^{\prime}=0
$$


we have directly

$$
U_{1 a}=-w_{0 b}^{\prime}
$$

and there is no advantage to be gained by computing $R^{0}=0, R_{0}=0$. But for $Z^{(0)}=0, Z_{(0)}=0$, we find

$$
\begin{aligned}
& w_{1 a}=-\frac{\sigma}{1-\sigma} U_{0 b}^{*}+\frac{(1+\sigma)(1-2 \sigma) \mathrm{S}^{0}}{(1-\sigma) E} \\
& w_{1 a}=-\frac{\sigma}{1-\sigma} U_{0 \ddot{b}}+\frac{(1+\sigma)(1-2 \sigma) \mathrm{S}_{0}}{(1-\sigma) E}
\end{aligned}
$$

and these results are more concisely given by $\mathrm{Z}_{0}=0$ and $\mathrm{Z}^{0}=0$, namely

$$
\begin{gathered}
S^{0} \equiv \mathrm{S}_{0}, \\
w_{1 a}=-\frac{\sigma}{1-\sigma} U_{0 b}^{*}+\frac{(1+\sigma)(1-2 \sigma) \mathrm{S}_{0}^{0}}{(1-\sigma) E},
\end{gathered}
$$

where $\mathrm{S}^{0} \equiv \mathrm{S}_{0} \equiv \mathrm{S}_{0}^{0}$. We note that a tension or pressure to be of as low order as $t^{0}$ must be the same on both bases. Indeed, we shall find that the difference between the applied tractions on the two bases is of order $t^{3}$, or else nil.

We turn next to $\mathrm{F}_{r}^{(0)}=0, \mathrm{~F}_{z}^{(0)}=0$, and find

$$
\begin{aligned}
& U_{2}=U_{2 b}+U_{2 a} \zeta-\frac{1}{2(1-2 \sigma)} w_{1 a}^{\prime} \zeta^{2}-\frac{1-\sigma}{1-2 \sigma} U_{0 b}^{* \prime} \zeta^{2}, \\
& w_{2}=w_{2 b}+w_{2 a} \zeta-\frac{1}{4(1-\sigma)} U_{1 a}^{*} \zeta^{2}-\frac{1-2 \sigma}{4(1-\sigma)} w_{0 b}^{\prime * *} \zeta^{2} .
\end{aligned}
$$

To simplify these results, we have at our disposal (31b) and (33b). If we further restrict $S_{0}^{0}$ (in general a function of $r$ ) to be a constant, $\dagger$ it follows from $(33 b)$ that

$$
u_{1 a}^{\prime}=-\frac{\sigma}{1-\sigma} U_{0 b}^{* \prime}
$$

† It should perhaps be remarked at this point that the restrictions imposed in the present paragraph are not such as to obscure the method of procedure in the general case. Our aim is not so much to be exhaustive in treatment as to cover adequately a number of sufficiently suggestive cases. 
and we may write (34) as follows:

$$
\begin{aligned}
& U_{2}=U_{2 b}+U_{2 a} \zeta-\frac{2-\sigma}{2(1-\sigma)} U_{0 b}^{* \prime} \zeta^{2}, \\
& w_{2}=w_{2 b}+w_{2 a} \zeta+\frac{\sigma}{2(1-\sigma)} w_{0}^{\prime *} \zeta^{*}:
\end{aligned}
$$

Turning again to the traction conditions, we findt for $R^{1}=0, R_{1}=0$

$$
\begin{gathered}
w_{1 b}^{\prime}+U_{2 a}=0 \\
U_{0 b \boldsymbol{\alpha}}^{* \prime}+\left[U_{0 b}^{\prime}+\sigma \frac{U_{0 b}}{r}\right] \alpha^{\prime}=\frac{(1+\sigma)(1-2 \sigma) \mathbf{S}_{0}^{0} \alpha^{\prime}}{E}
\end{gathered}
$$

By means of (37a) we eliminate $U_{2 a}$ once for all; $(37 b)$ is the first of a system of differential equations which we shall study separately in the following paragraph.

For $Z_{1}=0, Z^{1}=0$ the results are, respectively,

$$
\begin{gathered}
S^{1} \equiv \mathrm{S}_{1}, \\
w_{2 a}=-\frac{\sigma}{1-\sigma} U_{1 b}^{*}+\frac{(1+\sigma)(1-2 \sigma) \mathrm{S}_{1}^{1}}{(1-\sigma) E},
\end{gathered}
$$

where $\mathrm{S}^{1} \equiv \mathrm{S}_{1} \equiv \mathrm{S}_{1}^{1}$.

We here introduce a further restriction, $\ddagger$ and set $S_{1}^{1} \equiv 0$. Indeed, in prescribing the load we propose to consider only three cases of tractions applied to the faces of the plate, namely

$$
\mathrm{S}^{\prime \prime} \equiv \mathrm{S}_{\boldsymbol{v}} \equiv \mathbf{0}
$$

$$
\mathrm{S}^{\nu} \equiv \mathrm{S}_{\nu} \equiv \mathrm{S}_{0}^{0}
$$

$$
\mathrm{S}^{\nu} \equiv \mathrm{S}^{3} t^{3}, \mathrm{~S}_{\nu} \equiv \mathbf{0}
$$

$\uparrow$ We use (33), and the fact that $\mathbf{S}_{0}^{\mathbf{0}^{\prime}} \equiv 0$.

$\ddagger$ This is no restriction physically, for we are prescribing the load. 
This means that all the S's, that is, coefficients in (18), vanish identically save $S_{0}^{0}$ and $S^{8}$, and it will simplify the presentation to have these facts before us at the outset.

If $S_{1}^{1} \equiv 0$, we have, from $(38 b)$,

$$
w_{2 a}=-\frac{\sigma}{1-\sigma} U_{1 b}^{*}
$$

and recalling ( $37 a$ ), it is clear that both $U_{2 a}$ and $w_{2 a}$ may now be eliminated once for all. Bearing this fact in mind, and making use of (35), we have for $\mathrm{F}_{r}^{(1)}=0, \mathrm{~F}_{z}^{(1)}=0$ the following simplified forms:

$$
\begin{aligned}
& U_{3}=U_{3 b}+U_{8 a} \zeta-\frac{2-\sigma}{2(1-\sigma)} U_{1 b}^{* \prime} \zeta^{2}+\frac{2-\sigma}{6(1-\sigma)} w_{0 b}^{\prime \prime \prime} \zeta^{3} \\
& w_{3}=w_{3 b}+w_{3 a} \zeta+\frac{\sigma}{2(1-\sigma)} w_{1 b}^{\prime *} \zeta^{2}+\frac{1+\sigma}{6(1-\sigma)} U_{0 b}^{* \prime *} \zeta^{3} .
\end{aligned}
$$

Finally we note also that $Z_{2}=0$ gives $S^{2} \equiv S_{2}$, using (37a), and we set $\mathrm{S}^{2} \equiv \mathrm{S}_{2} \equiv \mathrm{S}_{2}^{2} \equiv 0$.

In this paragraph we consider no further traction equations; indeed, the later differential equations become rather involved, and we devote a separate paragraph to their systematic treatment. We have attained our object; namely, to obtain in the formulas of displacement such simplifications as are possible without restriction upon the applications. Aside, then, from the traction conditions already employed, we confine ourselves to body force equations only, and thus obtain general forms for $U$ and $w$ that satisfy (14) formally. These play so important a rôle that we record them below through the terms in $t^{6}$ :

$$
\begin{aligned}
& \text { (41a) } \quad U=U_{0 b}+\left[U_{1 b}-w_{0 b}^{\prime} \zeta\right] t+\left[U_{2 b}-w_{1 b}^{\prime} \zeta-\frac{2-\sigma}{2(1-\sigma)} U_{0 b}^{* \prime} \zeta^{2}\right] t^{2} \\
& +\left[U_{3 b}+U_{3 a} \zeta-\frac{2-\sigma}{2(1-\sigma)} U_{1 b}^{* \prime} \zeta^{2}+\frac{2-\sigma}{6(1-\sigma)} w_{0 b}^{\prime \prime \prime} \zeta^{3}\right] t^{3}+\left[U_{4 b}+U_{4 a} \zeta\right. \\
& -\frac{1}{2(1-2 \sigma)} \omega_{3 a}^{\prime} \zeta^{2}-\frac{1-\sigma}{1-2 \sigma} U_{2 b}^{* \prime} \zeta^{2}+\frac{2-\sigma}{6(1-\sigma)} w_{1 b}^{\prime \prime \prime} \zeta^{3} \\
& \left.+\frac{3-\sigma}{24(1-\sigma)} U_{0 b}^{* \prime \prime \prime} \zeta^{4}\right] t^{4}+\left[U_{5 b}+U_{5 a} \zeta-\frac{1}{2(1-2 \sigma)} w_{4 a} \zeta^{2}\right.
\end{aligned}
$$


1923]

$$
\begin{aligned}
& -\frac{1-\sigma}{1-2 \sigma} U_{8 b}^{* \prime} \zeta^{2}-\frac{3-2 \sigma}{12(1-\sigma)} U_{8 a}^{* \prime} \zeta^{8}+\frac{1}{12(1-\sigma)} w_{2 b}^{\prime * \prime} \zeta^{3} \\
& \left.+\frac{3-\sigma}{24(1-\sigma)} U_{1 b}^{* * \prime \prime} \zeta^{4}-\frac{3-\sigma}{120(1-\sigma)} w_{0 b}^{\prime * * \prime} \zeta^{5}\right] t^{5} \\
& +\left[U_{b b}+U_{6 a} \zeta-\frac{1}{2(1-2 \sigma)} w_{b a}^{\prime} \zeta^{2}-\frac{1-\sigma}{1-2 \sigma} U_{4 b}^{* \prime} \zeta^{2}-\frac{3-2 \sigma}{12(1-\sigma)} U_{4 a}^{* \prime} \zeta^{3}\right. \\
& +\frac{1}{12(1-\sigma)} w_{B b}^{\prime * \prime} \zeta^{3}+\frac{1}{12(1-2 \sigma)} w_{8 a}^{\prime * \prime} \zeta^{4}+\frac{3-2 \sigma}{24(1-2 \sigma)} U_{2 b}^{*{ }^{\prime * \prime}} \zeta^{4} \\
& \left.-\frac{3-\sigma}{120(1-\sigma)} w_{1 b}^{\prime \prime \prime * \prime} \zeta^{5}-\frac{4-\sigma}{720(1-\sigma)} U_{0 b}^{* \prime \prime \prime * \prime} \zeta^{6}\right] t^{6}+\cdots ;
\end{aligned}
$$

(41b) $\quad w=w_{0 b}+\left[w_{1 b}+w_{1 a} \zeta\right] t+\left[w_{2 b}-\frac{\sigma}{1-\sigma} U_{1 b}^{*} \zeta+\frac{\sigma}{2(1-\sigma)} w_{0 b}^{\prime *} \zeta^{2}\right] t^{2}$

$$
+\left[w_{8 b}+w_{3 a} \zeta+\frac{\sigma}{2(1-\sigma)} w_{1 b}^{\prime *} \zeta^{2}+\frac{1+\sigma}{6(1-\sigma)} U_{0 b}^{* *} \zeta^{3}\right] t^{3}+\left[w_{4 b}+w_{4 a} \zeta\right.
$$$$
-\frac{1}{4(1-\sigma)} U_{8 a}^{*} \zeta^{2}-\frac{1-2 \sigma}{4(1-\sigma)} w_{2 b}^{\prime *} \zeta^{2}+\frac{1+\sigma}{6(1-\sigma)} U_{1 b}^{* * *} \zeta^{3}
$$$$
\left.-\frac{1+\sigma}{24(1-\sigma)} w_{0 b}^{\prime \prime \prime *} \zeta^{4}\right] t^{4}+\left[w_{5 b}+w_{5 a} \zeta-\frac{1}{4(1-\sigma)} U_{4 a}^{*} \zeta^{2}\right.
$$$$
\frac{1-2 \sigma}{4(1-\sigma)} w_{8 b}^{\prime *} \zeta^{z}+-\frac{\sigma}{3(1-2 \sigma)} w_{8 a}^{\prime *} \zeta^{3}+\frac{1}{6(1-2 \sigma)} U_{2 b}^{* *} \zeta^{3}
$$$$
\left.-\frac{1+\sigma}{24(1-\sigma)} w_{1 b}^{\prime * * *} \zeta^{4}-\frac{2+\sigma}{120(1-\sigma)} U_{0 b}^{* * * *} \zeta^{5}\right] t^{5}+\left[w_{b b}+w_{b a} \zeta\right.
$$$$
-\frac{1}{4(1-\sigma)} U_{b a}^{*} \zeta^{2}-\frac{1-2 \sigma}{4(1-\sigma)} w_{4 b}^{\prime *} \zeta^{2}+\frac{\sigma}{3(1-2 \sigma)} w_{4 a}^{\prime *} \zeta^{3}
$$$$
+\frac{1}{6(1-2 \sigma)} U_{B b}^{* \prime *} \zeta^{8}+\frac{1}{24(1-\sigma)} U_{B a}^{* \prime *} \zeta^{4}-\frac{\sigma}{24(1-\sigma)} w_{2 b}^{\prime \prime \prime *} \zeta^{4}
$$$$
\left.-\frac{2+\sigma}{120(1-\sigma)} U_{1 b}^{* \prime * \prime *} \zeta^{5}+\frac{2+\sigma}{720(1-\sigma)} w_{0 b}^{\prime * \prime * \prime *} \zeta^{6}\right] t^{6}+\cdots
$$ 
4. Four systems of differential equations obtained from the surface traction conditions. In the preceding paragraph, the surface traction equations (30) were disposed of for the cases $n=-1$ and $n=0$; we made use also of the equations $R^{1}=0, Z^{1}=0, Z_{1}=0, Z_{2}=0$. The remaining differential equations are considered in the present paragraph.

The differential equations (30) are total, $r$ being the independent variable. Furthermore, the equations are linear, with coefficients that are functions of $\alpha$, and for plates of uniform thickness these coefficients are obviously constants. The functions of $r$ that are arbitrary in (41) are determined by these differential equations up to the constants of integration.

When the surface traction differential equations are written out in full, it is found that they fall naturally into four systems according as they involve the unknowns $U_{0 b}, U_{1 b}, w_{0 b}, w_{1 b}$. The facts with reference to the equations and unknowns which enter in these four systems are given in the following table:

\begin{tabular}{|c|c|c|c|}
\hline System & Equations & $\begin{array}{c}\text { Functions } \\
n=1,2, \ldots, m .\end{array}$ & $\begin{array}{c}\text { Number } \\
\text { of equations } \\
\text { (or functions) } \\
m=0,1,2, \ldots\end{array}$ \\
\hline$U_{0 b}$ & $\begin{array}{l}\mathrm{R}_{2 n+1}=0, n=0,1,2, \ldots, m ; \\
\mathrm{Z}^{2 n}=0, n=1,2, \ldots, m .\end{array}$ & $\begin{array}{c}U_{0 b} \\
U_{2 n, b} ; w_{2 n+1, a} .\end{array}$ & $2 m+1$ \\
\hline$U_{1 b}$ & $\begin{array}{l}\mathrm{R}_{2 n+2}=0, n=0,1,2, \ldots, m ; \\
\mathrm{Z}^{2 n+1}=0, n=1,2, \ldots, m .\end{array}$ & $\begin{array}{c}U_{1 b} \\
U_{2 n+1, b} ; w_{2 n+8, a} .\end{array}$ & $2 m+1$ \\
\hline$w_{v b}$ & $\begin{array}{l}\mathrm{R}^{2 n+2}=0 \\
\mathrm{Z}_{2 n+3}=0,\end{array} \quad n=0,1,2, \ldots, m$. & $\begin{array}{c}w_{0 u} ; w_{2 m+2, b}^{\prime}+U_{2 m+s, a} \\
w_{2 n, b} ; U_{2 n+1, a}\end{array}$ & $2 m+2$ \\
\hline$w_{16}$ & $\begin{array}{l}\mathrm{R}^{2 n+3}=0, \\
\mathrm{Z}_{2 n+4}=0,\end{array}$ & $\begin{array}{c}w_{1 b} ; w_{2 m+9, b}^{\prime}+U_{2 m+d, a} \\
w_{2 n+1, b} ; U_{2 n+2, a}\end{array}$ & $2 m+2$ \\
\hline
\end{tabular}

The meaning of the table is best understood after a few of the differential equations of each system have been written out in full. For this purpose, and for the more important applications as well, it will suffice to consider only $m=0,1$. 
(a) THE $U_{\mathrm{vb}}$ SYSTEM

We have already met the first differential equation of the $U_{0 b}$ system. It is $R_{1}=0$, an equation which we found it convenient to compute while we were considering $\mathrm{R}^{1}=0$. We begin, then, with $(37 b)$ :

$$
U_{0 b}^{* \prime} \alpha+\left[U_{0 b}^{\prime}+\sigma \frac{U_{0 b}}{r}\right] \alpha^{\prime}=\frac{(1+\sigma)(1-2 \sigma) S_{0}^{0} \alpha^{\prime}}{E} .
$$

For $m=1$, we have to compute the equations $Z^{2}=0, R_{3}=0$. Since $\mathrm{S}_{2}^{2} \equiv 0$, we find, for $\mathrm{Z}^{2}=0$,

$$
\frac{1-2 \sigma}{1-\sigma}\left(U_{0 b}^{* \prime} \alpha^{2}\right)^{*}+2 \sigma U_{2 b}^{*}+2(1-\sigma) v_{3 a}=0
$$

where, by formula $(10 a)$,

$$
\left(U_{0 b}^{* \prime} \alpha^{2}\right)^{*}=U_{0 b}^{* * *} \alpha^{2}+2 U_{0 b}^{* \prime} \alpha \alpha^{\prime}
$$

The equation $R_{3}=0$, when written out in full, becomes

$$
\begin{gathered}
\frac{2(1-2 \sigma)}{3(1-\sigma)} U_{0 b}^{* \prime * \prime} \alpha^{3}+\frac{\sigma(1-2 \sigma)}{1-\sigma} U_{0 b}^{* *} \alpha^{2} \alpha^{\prime}+\frac{(2-\sigma)(1-2 \sigma)}{1-\sigma} U_{0 b}^{* \prime \prime} \alpha^{2} \alpha \\
-2(1-\sigma) U_{2 b}^{* \prime} \alpha-2 \sigma\left(U_{2 b}^{*}+w_{8 a}\right) \alpha^{\prime}-2(1-2 \sigma) U_{2 b}^{\prime} \alpha^{\prime} \\
-2 \sigma v_{3 a}^{\prime} \alpha=0 .
\end{gathered}
$$

The equations (43a) and (44) determine $U_{2 b}$ and $w_{3 a}$, since the function $U_{0 b}$ is known by (42). Without writing down the next two equations of the system, it is clear that they will involve the three functions already obtained and two new functions, $U_{4 b}$ and $w_{5 a}$, which they will determine.

(b) THE $U_{1 b}$ SYSTEM

Although the differential equations of this system may be readily obtained from those of the $U_{0 b}$ system by merely advancing all the indices by 1 , their importance in the applications to follow makes it desirable that they be explicitly set down at this point. (Also, the restrictions we have imposed upon the S's are going to alter the appearance of our equations somewhat, in 
particular the first three, so that the rule which advances indices is not easily applied.)

The first equation ( $m=0$ ) is $R_{2}=0$. Since $S_{1}^{1} \equiv 0$, we find

$$
U_{1 b}^{* \prime} \alpha+\left[U_{1 b}^{\prime}+\sigma \frac{U_{1 b}}{r}\right] \alpha^{\prime}=0
$$

When $m=1$, the equations are $Z^{3}=0$ and $R_{4}=0$; or

$$
\frac{1-2 \sigma}{1-\sigma}\left(U_{1 b}^{* \prime} \alpha^{2}\right)^{*}+2 \sigma U_{8 b}^{*}+2(1-\sigma) w_{4 a}=\frac{(1+\sigma)(1-2 \sigma)\left(S^{3}+S_{3}\right)}{E}
$$

and

$$
\begin{gathered}
\frac{2(1-2 \sigma)}{3(1-\sigma)} U_{1 b}^{* * \prime} \alpha^{3}+\frac{\sigma(1-2 \sigma)}{1-\sigma} U_{1 b}^{* \prime *} \alpha^{2} \alpha^{\prime}+\frac{(2-\sigma)(1-2 \sigma)}{1-\sigma} U_{b b}^{* \prime \prime} \alpha^{8} \alpha^{\prime} \\
-2(1-\sigma) U_{3 b}^{* \prime} \alpha-2 \sigma\left(U_{8 b}^{*}+w_{4 a}\right) \alpha^{\prime}-2(1-2 \sigma) U_{8 b}^{\prime} \alpha^{\prime}-2 \sigma w_{4 a}^{\prime} \alpha \\
=\frac{(1+\sigma)(1-2 \sigma)\left(S^{3}+S_{8}\right) \alpha^{\prime}}{E}
\end{gathered}
$$

The last two equations determine $U_{3 b}$ and $w_{4 a}$, since (45) has determined $U_{1 b} . S^{3}$ and $S_{3}$ are in general distinct. We shall take $S_{3} \equiv 0$ in agreement with § 3. In that paragraph we pointed out a fact which is now established; namely, that if $S^{\prime \prime}$ and $S_{v}$ are distinct, their difference is of order $t^{3}$.

We note that the $U_{0 b}$ and $U_{1 b}$ systenis are formally the same; if, in particular, the surface tractions on the bases are nil, the corresponding unknowns of the two systems differ only in the constants of integration.

(c) THE $w_{0 b}$ SYSTEM

For $m=0$, the table gives two differential equations of the $w_{0 b}$ system; namely, $R^{2}=0$ and $Z_{s}=0$. We have, in full,

$$
\begin{aligned}
& \frac{1}{1-\sigma} w_{0 b}^{\prime * \prime} \alpha^{2}+\frac{2}{1-\sigma}\left[w_{0 b}^{\prime \prime}+\sigma \frac{u_{0 b}^{\prime}}{r}\right] \alpha \alpha^{\prime}+\left(w_{2 b}^{\prime}+U_{8 a}\right)=0 \\
& {\left[\frac{1}{3(1-\sigma)} w_{0 b}^{\prime * \prime} \alpha^{3}+\left(w_{2 b}^{\prime}+U_{8 a}\right) \alpha\right]^{*}=-\frac{(1+\sigma)\left(S^{3}-S_{8}\right)}{E}}
\end{aligned}
$$


These equations determine $w_{0 b}$ and $w_{2 b}^{\prime}+U_{3 a}$, if we admit as a single unknown the combination $w_{2 b}^{\prime}+U_{8 a}$. It will be found that the next two equations, $m=1$, determine $w_{2 b}$ and $U_{3 a}$ individually. Since we are interested in $w_{0 b}$, we eliminate $w_{2 b}^{\prime}+U_{3 a}$ from (48) and (49) and obtain the following differential equation for the determination of $w_{0 b}$ (taking $S_{3} \equiv 0$ ):

$$
\left[w_{0 b}^{\prime \prime \prime} \alpha^{8}+3\left(w_{0 b}^{\prime \prime}+\sigma \frac{w_{0 b}^{\prime}}{r}\right) \alpha^{2} \alpha^{\prime}\right]^{*}=\frac{3\left(1-\sigma^{2}\right) S^{3}}{2 E}
$$

For $m=1$, the equations are $\mathrm{R}^{4}=0, \mathrm{Z}_{5}=0$; or

$$
\begin{aligned}
& \frac{1}{6(1-\sigma)} w_{0 b}^{\prime * * *} \alpha^{4}+\frac{1}{3(1-\sigma)}\left[\sigma w_{0 b}^{\prime * * *}(2-\sigma) w_{0 b}^{\prime * \prime \prime}\right] \alpha^{3} \alpha^{\prime} \\
+ & \frac{1}{2(1-\sigma)}\left[(2-\sigma) U_{s a}^{* \prime}-\sigma w_{2 b}^{\prime * \prime}\right] \alpha^{2}+\frac{\sigma}{1-\sigma}\left[U_{8 a}^{*}-w_{2 b}^{\prime *}\right] \alpha \alpha^{\prime} \\
+ & 2 U_{8 a}^{\prime} \alpha \alpha^{\prime}-\left(w_{4 b}^{\prime}+U_{5 a}\right)=\frac{(1+\sigma)\left(S^{3}-S_{3}\right) \alpha^{\prime}}{E}
\end{aligned}
$$

and, since we take $\mathrm{S}^{6} \equiv \mathrm{S}_{5} \equiv 0$,

$$
\begin{aligned}
\left\{\frac{1}{30(1-\sigma)} w_{0 b}^{\prime \prime \prime \prime} \alpha^{5}+\right. & \frac{1}{6(1-\sigma)}\left[(2-\sigma) U_{3 a}^{* \prime}-\sigma w_{2 b}^{\prime \prime \prime}\right] \alpha^{3} \\
& \left.-\left(w_{4 b}^{\prime}+U_{b a}\right) \alpha\right\}^{*}=0=\frac{(1+\sigma)\left(\mathrm{S}^{5}-\mathrm{S}_{5}\right)}{E} .
\end{aligned}
$$

From these last two equations we can eliminate $w_{4 b}^{\prime}+U_{5 a}$, and obtain an equation involving $w_{0 b}, w_{2 b}$ and $U_{8 a}$; this equation, together with (48), will determine $w_{2 b}$ and $U_{8 a}$ individually, $w_{0 b}$ having been determined by (50). The foregoing is typical of the general stage in the computation. Let the case $m=2$ be a final illustration: we obtain two equations from which to eliminate $w_{6 b}^{\prime}+U_{7 a}$, and are thus led to a single equation in $w_{4 b}$ and $U_{5 a}$ which, together with (51), determines these two functions.

(d) THE $w_{1 b}$ SYSTEM

This system of differential equations is obtained at once from the $w_{0 b}$ system by advancing all indices by 1 . Since we have $\mathrm{S}_{2}^{2} \equiv \mathrm{S}^{4} \equiv \mathrm{S}_{4} \equiv \ldots \equiv 0$, the 
right-hand members are all 0 . In all other respects the equations are those of the $w_{0 b}$ system with the indices advanced by 1 , and it is quite unnecessary to write them out in full.

Since the $U_{1 b}$ and $w_{1 b}$ systems are in form the same as the $U_{0 b}$ and $w_{0 b}$ systems, we see that there are essentially only two distinct types of systems of surface traction differential equations. We shall see later $(\S 6)$ how these two systems are related to the two modes of displacement, radial and axial. With reference to the structure of the differential equations of the two systems, note that when we set $U_{0 b} \equiv 0, w_{c b} \equiv 0$, and decrease all indices by 2 , we get back again to the original $U_{0 b}$ and $w_{0 b}$ systems. In this connection, we recall the fact that we are dealing with an elastic theory that admits of superposition of solutions.

The striking fact that emerges from the situation just described is that all the differential equations of a system have the same "reduced" linear homogeneous equation. For example, in the $w_{0 b}$ system, the function $w_{2 b}$ is determined by a differential equation which is found, when computed, to have the same corresponding reduced equation as (50), and similarly for $v_{4 b}$, and so on. This property of the system suggests that once a method is found for integrating the leading equation, all subsequent equations of the system (although the non-homogeneous members become more and more involved) may possibly be integrable by the same method.

It is also interesting to note how closely the reduced equation for the system of axial typet resembles the reduced equation for the system of radial type. Indeed, these reduced equations may be written respectively in the forms

$$
\begin{aligned}
& y^{* \prime}+3\left(y^{\prime}+\sigma \frac{y}{r}\right) \frac{\alpha^{\prime}}{\alpha}=0, \\
& y^{* \prime}+\left(y^{\prime}+\sigma \frac{y}{r}\right) \frac{\alpha^{\prime}}{\alpha}=0 .
\end{aligned}
$$

Although we have verified the table for the values $m=0,1$ only, much light has been shed on the nature of the differential equations, and, incidentally, we have obtained a sufficient number of these equations to enable us to meet the immediate requirements of the applications.

5. Boundary conditions at an edge. In (41), we have forms for $U$ and $w$ that satisfy the body force conditions formally, and when the $U_{a}$ 's, $U_{b}$ 's, $w_{a}$ 's, and $w_{b}$ 's satisfy the differential equations of $\S 4$, these forms for $U$ and $w$ also meet the requirements imposed by the surface traction conditions. To complete the program outlined at the beginning of $\S 3$, it remains to determine the constants of integration which enter in the solutions of the differential

\footnotetext{
$\dagger$ See § 6.
} 
equations. When this has been accomplished by means of the boundary conditions at the edge (or edges) of the plate, $U$ and $w$ are uniquely determined and the particular problem in hand is formally solved. (We shall consistently use the expression "boundary conditions" to refer to boundary conditions at an edge.)

In dealing with boundary conditions, we adopt the conventions of Love, Chapter XXII. For circular plates, an "edge-line" is the intersection of the "middle plane" ( $z=0)$ with a circular cylinder $\left(r=r_{0}\right)$ whose axis is the axis of the plate. The componentst of the stress-resultant are denoted by $T, N$, and the stress-couple is denoted by $G$. The directions of these components and the conventions with regard to sensef are illustrated in Fig. 1. If the normal $v$ is drawn outwards, we are considering the action of the portion of the plate exterior to $r=r_{0}$ upon that part of the plate interior to $r=r_{0}$. A positive $T$ is a radial tension, and points in the direction of the increasing $r$; similarly, $N$ is directed positively in the sense of the increasing $z$, and denotes a shearing force normal to the middle plane. The couple $G$ is flexural.

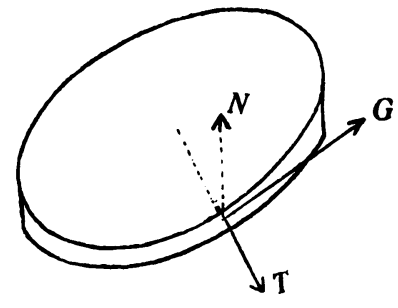

Fig. 1 .

The three stress-components are given by the expressions

$$
\begin{aligned}
& T=\int_{-a}^{a} t \hat{r} d \zeta, \\
& N=\int_{-a}^{a} t \hat{r z} d \zeta \\
& G=\int_{-a}^{a} t^{2} \widehat{r r} \zeta d \zeta,
\end{aligned}
$$

where the integrands are expressed in terms of $\zeta$ by (16); $U$ and $w$ are given by the series (41), and the $U_{a}$ 's, $U_{b}$ 's, $w_{a}$ 's and $w_{b}$ 's are given by the solutions of the differential equations of $\$ 4$. If the plate is thick, the traction components have prescribed values at every point of the edge (Love, p. 464); but since

† There is no vector couple in the $z$ direction, and for a circular plate, $S \equiv H \equiv 0$ (cf. Love, p. 56 and p. 141). Thus we are concerned at present only with $T, N G$.

$\ddagger$ Cf. Love, p. 461. 
we are considering thin plates (cf. $\S 16$ ), the tractions applied to the edge may be represented by their force- and couple-resultants estimated per unit of length of the edge-line.

We follow Love (p. 465) in expressing the statical equivalence of the applied tractions, T, N, G, and the stress-resultant and stress-couple components at an edge by equations of the type

$$
T=\mathrm{T}, \quad N=\mathrm{N}, \quad \theta=\mathrm{G} .
$$

Note that the right-hand members are total applied tractions. Assuming that integrations with regard to $\zeta$ have been performed, we write the applied traction as follows:

$$
\begin{aligned}
& \mathrm{T}=\mathrm{T}_{0}+\mathrm{T}_{1} t+\mathrm{T}_{2} t^{2}+\cdots, \\
& \mathrm{N}=\mathrm{N}_{0}+\mathrm{N}_{1} t+\mathrm{N}_{2} t^{2}+\cdots, \\
& \mathrm{G}=\mathrm{G}_{0}+\mathrm{G}_{1} t+\mathrm{G}_{2} t^{2}+\cdots,
\end{aligned}
$$

where the coefficients are functions of $r$ only, and using $(16 a)$ and $(16 e)$, we see that relations (54) are identities in $t$ of the form

(56a)

$$
\frac{E}{(1+\sigma)(1-2 \sigma)} \int_{-a}^{a}\left\{\sigma\left(U^{*} t+\frac{\partial w}{\partial \zeta}\right)+(1-2 \sigma) U^{\prime} t\right\} d \zeta \equiv \mathrm{T}
$$

$$
\begin{aligned}
& \frac{E}{2(1+\sigma)} \int_{-a}^{a}\left\{\frac{\partial U}{\partial \zeta}+w^{\prime} t\right\} d \zeta \equiv \mathrm{N} \\
& \frac{E}{(1+\sigma)(1-2 \sigma)} \int_{-\alpha}^{a}\left\{\sigma\left(U^{*} t+\frac{\partial w}{\partial \zeta}\right)+(1-2 \sigma) U^{\prime} t\right\} t \zeta d \zeta \overline{\bar{t}} \mathrm{G}
\end{aligned}
$$

Upon equating coefficients of like powers of $t$, we obtain equations which determine the constants of integration, provided the right-hand members are known at the edgets. If the displacements are prescribed at the edges, so that the constants of integration are already known, our equations enable us to compute $\mathbf{T}, \mathrm{N}, \mathrm{G}$ as functions of $r$ of the form (55). 
We give below the definitions of certain types of boundary conditions which may obtain at an edge $r=r_{0}$ :

(i) At a "free" edge the requirements are

$$
T\left(r_{0}\right)=N\left(r_{0}\right)=G\left(r_{0}\right)=0 .
$$

(ii) At a "supported" edge the axial displacement of a point on the middle plane (at the edge-line) is nil, but there is no restriction on the radial displacement of such a point. Also, $T$ and $G$ vanish, so that the complete conditions at a supported edge are

$$
\because\left(r_{0}, 0\right)=T\left(r_{0}\right)=G\left(r_{0}\right)=0 .
$$

(iii) At a "clamped" edge we have

$$
U\left(r_{0}, 0\right)=w\left(r_{0}, 0\right)=w^{\prime}\left(r_{0}, 0\right)=0 ;
$$

in short, there is neither axial nor radial displacement of a point on the edgeline, and the inclination of the middle plane is not permitted to vary.

To complete our list, we note a type of boundary condition which enters in $\S \S 7,8$. There both edges of an incomplete plate are subject to radial pressure (or tension), and the boundary conditions take the form

$$
T\left(r_{0}\right)=\mathrm{T}\left(r_{0}\right), \quad T\left(r_{1}\right)=\mathrm{T}\left(r_{1}\right)
$$

The applications will require that we have on record a few of the equations obtained from (56) by equating coefficients of like powers of $t$. From (56a) we find $\mathrm{T}_{0} \equiv 0$. From the terms in $t$ we have

$$
\frac{2 \alpha E}{1-\sigma^{2}}\left[U_{0 b}^{\prime}+\sigma \frac{U_{0 b}}{r}\right]+\frac{2 \sigma \alpha}{1-\sigma} \mathrm{S}_{0}^{0}=\mathrm{T}_{1}
$$

and we note that $\mathrm{T}$ is of order $t$. Equating the terms in $t^{2}$ gives the same result that is obtained by advancing all indices by 1 in $(61 a)$; namely

$$
\frac{2 \alpha E}{1-\sigma^{2}}\left[U_{1 b}^{\prime}+\sigma \frac{U_{1 b}}{r}\right]=\mathrm{T}_{2} .
$$


In computing the equations from the terms in $t^{3}$ and $t^{4}$, the integrations with regard to $\zeta$ should be carried out, and it is also useful to eliminate $w_{3 a}$ and $w_{4 a}$ by means of (43a) and (46). Although these formulas simplify for plates of constant thickness, the general forms are rather forbidding; we have

$(61 c)$

$$
\frac{2 \alpha E}{1-\sigma^{2}}\left[U_{2 b}^{\prime}+\sigma \frac{U_{2 b}}{r}-\frac{\alpha}{6(1-\sigma)}\left\{\sigma(4-\sigma) U_{0 b}^{* \prime *} \alpha\right.\right.
$$

$$
\frac{2 \alpha E}{1-\sigma^{8}}\left[U_{3 b}^{\prime}+\sigma \frac{U_{8 b}}{v}-\frac{\alpha}{6(1-\sigma)}\left\{\sigma(4-\sigma) U_{1 b}^{* * *} \alpha\right.\right.
$$

$$
\left.\left.+6 \sigma U_{1 b}^{* \prime} \alpha^{\prime}+(2-\sigma)(1-\sigma) U_{1 b}^{* \prime \prime} \alpha\right\}\right]+\frac{\sigma \alpha \mathrm{S}^{3}}{1-\sigma}=\mathrm{T}_{4} .
$$

Note in $(61 c)$ that $U_{0 b}$ is known, so that the essential relationship is between the functions $U_{2 b}$ and $\mathrm{T}_{3} ;$ a similar remark applies to $(61 d)$. Recall that $w_{8 a}$ and $w_{4 a}$ are known in terms of $U_{2 b}$ and $U_{3 b}$, respectively.

From (56b), we find $\mathrm{N}_{0} \equiv \mathrm{N}_{1} \equiv \mathrm{N}_{2} \equiv 0$. Equating terms in $t^{3}$, we have

$$
w_{0 b}^{\prime * \prime} \alpha^{3}+3\left[w_{0 b}^{\prime \prime}+\sigma \frac{w_{0 b}^{\prime}}{r}\right] \alpha^{2} \alpha^{\prime}=-\frac{3\left(1-\sigma^{2}\right) N_{3}}{2 E} .
$$

If the plate is of constant thickness $\left(\alpha=\eta, \alpha^{\prime}=0\right)$, the result is

$$
w_{0 b}^{\prime * \prime}=-\frac{\mathrm{N}_{3}}{\delta}
$$

where

$$
\delta=\frac{2 E \eta^{3}}{3\left(1-\sigma^{2}\right)}
$$

or, eliminating $t$ by means of the relationst $h=\eta t, D=\delta t^{8}$, we may write

$$
D=\frac{2 E h^{3}}{3\left(1-\sigma^{2}\right)}
$$

† These relations are in agreement with the notation which we first introduced when we wrote $z=\zeta t$ rather than $z=z t$. The Greek letter notation seems to conduce to clearness. 
where $2 h$ is the actual thickness of the plate and $D$ is the "flexural rigidity" (Love, p. 470).

The equation in $\mathrm{N}_{4}$ is obtained at once from $(62 a)$ by advancing the indices by 1. Equating terms in $t^{5}$, we find (retaining only even powers of $\zeta$ in the integrand)

$$
\begin{aligned}
\frac{E}{2(1+\sigma)} \int_{-a}^{a}\left\{-\frac{1}{6(1-\sigma)} w_{0 b}^{\prime * * \prime} \zeta^{4}+-\frac{\sigma}{2(1-\sigma)} w_{2 b}^{\prime * \prime} \zeta^{2}\right. \\
\left.-\frac{2-\sigma}{2(1-\sigma)} U_{3 a}^{* \prime} \zeta^{2}+w_{4 b}^{\prime}+U_{5 a}\right\} d \zeta=\mathrm{N}_{5} .
\end{aligned}
$$

From this equation we can eliminate $w_{4 b}^{\prime}+U_{5 a}$ by means of (51) and $U_{3 a}$ by means of (48); and integrating, we obtain a relation between $w_{2 b}$ and $N_{5}$, since $w_{0 b}$ is already known. Since the relation is rather involved, we shall be content to write it out only for the special case $\alpha^{\prime}=0 ;(62 e)$ then reduces to

$$
w_{2 b}^{\prime * \prime}-\frac{3 \sigma-8}{10(1-\sigma)} w_{0 b}^{\prime * * \prime} \eta^{8}=-\frac{\mathrm{N}_{5}}{\delta}
$$

We turn finally to $(56 c)$ and find $G_{0} \equiv G_{1} \equiv G_{2} \equiv 0$; it appears that $G$, as well as $\mathrm{N}$, must be of order $t^{3}$. We find

$$
\left[w_{0 b}^{\prime \prime}+\sigma \frac{w_{0 b}^{\prime}}{r}\right] \alpha^{3}=-\frac{3\left(1-\sigma^{2}\right)}{2 E} G_{3}
$$

and a similar equation in $\mathrm{G}_{4}$ obtained by advancing indices by 1 .

The terms in $t^{5}$ give us $(63 b)$

$$
\begin{aligned}
\frac{E}{1+\sigma} \int_{-a}^{a}\left\{\frac{\sigma}{6(1-\sigma)}\left[w_{0 b}^{\prime * *} \zeta^{3}-3 w_{2 b}^{\prime *} \zeta+3 U_{3 a}^{*} \zeta\right]\right. \\
\left.+\left[\frac{2-\sigma}{6(1-\sigma)} w_{0 b}^{* \prime \prime} \zeta^{3}+U_{8 a}^{\prime} \zeta\right]\right\} \zeta d \zeta=\mathrm{G}_{3}
\end{aligned}
$$

and when we eliminate $U_{3 a}$ by means of (48) and integrate, we have the desired relation between $w_{2 b}$ and $G_{5}$. If $\alpha^{\prime}=0$, we find

$(63 c) w_{2 b}^{\prime \prime}+\sigma \frac{w_{2 b}^{\prime}}{r}+\frac{\eta^{2}}{10(1-\sigma)}\left[\sigma(4+\sigma) w_{0 b}^{\prime * *}+(1-\sigma)(8+\sigma) w_{0 b}^{\prime * \prime \prime}\right]=-\frac{G_{5}}{\delta}$ 
We have obtained enough of the relations of type (56) to enable us to deal with the simpler applications. If ' $T$ ' N, G are initially prescribed, the series (5う) are either nil or else terminate with the leading term; for example: $\mathrm{T} \equiv \mathrm{T}_{1} t$, $\mathrm{N} \equiv \mathrm{N}_{3} t^{3}, \mathrm{G} \equiv \mathrm{G}_{3} t^{3}$. When these components are unknown elements and the plate is of constant thickness, the series (55), as well as (6), will terminate provided the distribution of load meets the requirements of $\S 13$. But for a random type of loading, or in the case of an initially prescribed central deflection, and obviously in cases of variable thickness, T, N, G, as well as $U, w$, are given in general by infinite series.

6. Analysis of the formulas of displacement by the aid of certain transformations. The formulas $(41)$ for $U$ and $w$, together with the differential equations of $\S 4$, are quite general; they enable us to handle, for example, a problem involving simultaneous radial and axial pressure. Before dealing with the applications, it is important to ascertain what simplifications occur in (41) when we consider

(i) the case of radial pressure only,

(ii) the case of axial pressure only.

In this connection, we make use of the following transformations:

$$
\begin{aligned}
& (t,-t),(w,-w), \\
& (\zeta,-\zeta),(u,-w),(\boldsymbol{\alpha},-\boldsymbol{\alpha}), \\
& (t,-t),(U,-U), \\
& (\zeta,-\zeta),(U,-U),(\boldsymbol{\alpha},-\boldsymbol{\alpha}),
\end{aligned}
$$

where $(t,-t)$ signifies the replacement of $t$ by $-t$, etc.

Clearly each of these transformations turns the plate upside down and at the same time reverses the signature of either $w$ or $U$. When one of these transformations does not disturb the conditions that characterize a given problem, it cannot influence the solution, and hence may be used to simplify the formulas of displacement.

If the transformations (64) are to leave $U$ and $w$ unchanged, they must leave unaltered the fundamental relations (14), (21), (56) which determine $U$ and $w . \dagger$ Since $F_{r} \equiv F_{z} \equiv 0$, all requirements are met in the case of (14).

\footnotetext{
† We need not concern ourselves here with the fact that the displacements themselves may be prescribed at the edges, as in (59). In such cases we discern by direct examination of (64) the transformations which do not disturb the boundary conditions.
} 
If (21) and (56) are to remain unchanged under (64), we find the following restrictions on $\mathrm{S}, \mathrm{T}, \mathrm{G}, \mathrm{N}$, where $\mathrm{S}$ refers to both $\mathrm{S}^{\nu}$ and $\mathrm{S}_{\boldsymbol{v}}: t$

$$
\begin{aligned}
& \mathrm{S}(t)=\mathrm{S}(-t), \quad \mathrm{T}(t)=-\mathrm{T}(-t) \\
& \mathrm{N}(t)=\mathrm{N}(-t), \mathrm{G}(t)=\mathrm{G}(-t)
\end{aligned}
$$

(65b) $\mathrm{N} \equiv \mathrm{G} \equiv 0$;

$$
\begin{aligned}
& \mathrm{S}(t)=-\mathrm{S}(-t), \quad \mathrm{T}(t)=\mathrm{T}(-t), \\
& \mathrm{N}(t)=-\mathrm{N}(-t), \mathrm{G}(t)=-\mathrm{G}(-t)
\end{aligned}
$$

$(65 d) \quad \mathrm{S} \equiv \mathrm{T} \equiv 0$.

Here the order of arrangement is the same as that in (64), and the conditions are concerned with the vanishing of certain terms in the expansions (18) and (55).

\section{(a) THe radial Case}

Under this heading we consider the pair of transformations (64a) and $(64 b)$, assuming that $(65 a)$ and $(65 b)$ do not conflict with the boundary conditions, so that $U$ and $w$ are unaltered. Using (6), we find that these transformations yield respectively the following relations:

$$
\begin{array}{lr}
U_{2 m+1}(r, \zeta) \equiv w_{2 m}(r, \zeta) \equiv 0 & (m=0,1,2, \ldots) \\
U_{m}(r, \zeta)-U_{m}(r,-\zeta) \equiv w_{m}(r, \zeta)+w_{m}(r,-\zeta) \equiv 0 \\
\\
\end{array}
$$

Turning to (41), we observe that (66a) implies the vanishing of all the unknowns of the $w_{0 b}$ and $U_{1 b}$ systems, and that $(66 \mathrm{~b})$ demands the vanishing of all the unknowns of the $w_{1 b}$ system; for (66) demands that each of these unknowns equal its negative, and since they are given uniquely by the differential equations of $\S 4$ (provided $(65 a)$ and $(65 b)$ do not conflict with the boundary conditions), this cannot happen except they vanish identically. With only the unknowns of the $U_{0 b}$ system remaining, the formulas (41) simplify tremendously. For future reference, the results are recorded below:

\footnotetext{
† In deriving $(65 b)$ and $(65 d)$, note that the right-hand members of (56) contain a multiplicative factor $2 a$ which changes sign under $(64 b)$ and $(64 d)$.
} 
(67a) $U=U_{0 b}+\left[U_{2 b}-\frac{2-\sigma}{2(1-\sigma)} U_{0 b}^{* \prime \prime} \zeta^{2}\right] t^{2}$

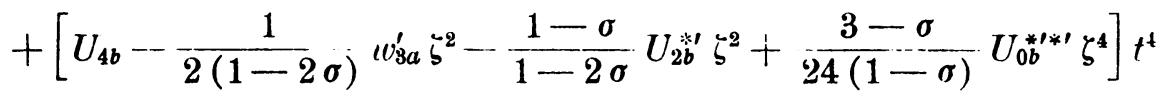

$$
\begin{aligned}
& +\left[U_{b b}-\frac{1}{2(1-2 \sigma)} w_{5 a}^{\prime} \zeta^{2}-\frac{1-\sigma}{1-2 \sigma} U_{4 b}^{* \prime} \zeta^{2}+\frac{1}{12(1-2 \sigma)} w_{B a}^{\prime * \prime} \zeta^{*}\right. \\
& \left.+\frac{3-2 \sigma}{24(1-2 \sigma)} U_{2 b}^{* \prime * \prime} \zeta^{4}-\frac{4-\sigma}{720(1-\sigma)} U_{0 b}^{* \prime * * * \prime} \zeta^{6}\right] t^{6}+\cdots,
\end{aligned}
$$

$$
\begin{aligned}
w=u_{1 a} \zeta t+ & {\left[u_{3 a} \zeta+\frac{1+\sigma}{6(1-\sigma)} U_{0 b}^{* *} \zeta^{3}\right] t^{3} } \\
& +\left[w_{5 a} \zeta+\frac{\sigma}{3(1-2 \sigma)} w_{3 a}^{* *} \zeta^{3}+\frac{1}{6(1-2 \sigma)} U_{2 b}^{* \prime *} \zeta^{3}\right. \\
& \left.-\frac{2+\sigma}{120(1-\sigma)} U_{0 b}^{* \prime * ; *} \zeta^{5}\right] t^{5}+\cdots
\end{aligned}
$$

Note that $w(r, 0)=0$; that is, the middle plane is stretched without deflection at any point. It is interesting to interpret physically the transformations $(64 a)$ and $(64 b)$; we observe that when $(t,-t)$ or $(\zeta,-\zeta)$, $(\alpha,-\alpha)$ turns the plate upside down, the requirement $(w,-w)$ takes care of the necessary reversal of sign in the axial displacement. The advantage of deducing formulas (67) at this point will appear when we arrive at the applications of $\S \S 7,8$.

\section{(b) The aXial case}

From (6), we find that the pair of transformations $(64 c)$ and $(64 d)$ yield respectively the relations

$$
\begin{aligned}
& U_{2 m}(r, \zeta) \equiv w_{2 m+1}(r, \zeta) \equiv 0 \\
& (m=0,1,2, \ldots) \\
& U_{m}(r, \zeta)+U_{m}(r,-\zeta) \\
& \equiv w_{m}(r, \zeta)-w_{m}(r,-\zeta) \equiv 0 \quad(m=0,1,2, \ldots) .
\end{aligned}
$$

By (41), the first of these relations implies the vanishing of all the unknowns of the $U_{0 b}$ and $w_{1 b}$ systems, and (68b) shows that all the unknowns of the $U_{1 b}$ system must vanish also. The argument here is the same as in the radial case. We are assuming, of course, that $(65 c)$ and $(65 d)$ do not conflict with the boundary conditions.

Before writing down any formulas of displacement for the axial case, let us see how much we can infer from $(64 d)$ when $\mathrm{S}^{\nu} \equiv \mathrm{S}^{3} t^{3} \neq 0, \mathrm{~S}_{\nu} \equiv 0$. 
Although the conditions of $(65 d)$ are no longer met, an examination of the differential equations of the $U_{1 b}$ system shows that the only unknowns of the system whose vanishing we cannot now infer are $U_{3 b}$ and $w_{4 a}$. Since the special case we are considering is one of vital importance in the applications, we shall retain these two unknowns in our formulas of displacement for the axial case.

The physical situation here is of considerable interest. When $U$ and $w$ are respectively even and odd functions of $\zeta$, as in the radial case, or respectively odd and even functions of $\zeta$, as in the puret axial case, there are obvious symmetrical relations governing the form into which an initially vertical filament is bent. But if the upper base of the plate is loaded, the terms in $U_{3 b}$ and $w_{4 a}$ enter, and $U$ and $u$ are no longer respectively odd and even functions of $\zeta$; not only are certain changes reflected in the form into which the initially vertical filament is bent, but also there is the new possibility that the middle plane is extended as well as bent.

The inclusion of the unknowns $U_{3 b}$ and $w_{4 a}$ gives us the following formulas of displacement in the axial case:

$$
\begin{aligned}
& U=-w_{0 b}^{\prime} \zeta t+\left[U_{3 b}+U_{8 a} \zeta+\frac{2-\sigma}{6(1-\sigma)} w_{0 b}^{\prime \prime \prime} \zeta^{3}\right] t^{3} \\
& +\left[U_{5 a} \zeta-\frac{1}{2(1-2 \sigma)} u_{4 a}^{\prime} \zeta^{2}-\frac{1-\sigma}{1-2 \sigma} U_{3 b}^{* \prime} \zeta^{2}-\frac{3-2 \sigma}{12(1-\sigma)} U_{8 a}^{* \prime} \zeta^{3}\right. \\
& \left.+\frac{1}{12(1-\sigma)} w_{2 b}^{\prime * \prime} \zeta^{3}-\frac{3-\sigma}{120(1-\sigma)} w_{0 b}^{\prime * \prime \prime \prime} \zeta^{5}\right] t^{5}+\cdots \\
& u=u_{0 b}+\left[w_{2 b}+\frac{\sigma}{2(1-\sigma)} w_{0 b}^{\prime *} \zeta^{2}\right] t^{2}+\left[w_{4 b}+w_{4 a} \zeta\right.
\end{aligned}
$$

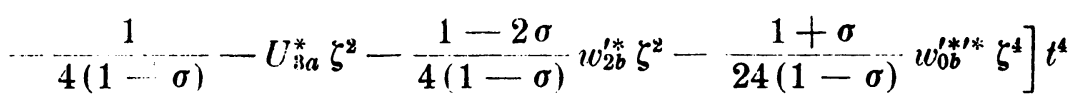

$$
\begin{aligned}
& +\left[u_{6 b}-\frac{1}{4(1-\sigma)} U_{5 a}^{*} \zeta^{2}-\frac{1-2 \sigma}{4(1-\sigma)} w_{4 b}^{*} \zeta^{2}+\frac{\sigma}{3(1-2 \sigma)} w_{4 a}^{\prime *} \zeta^{3}\right. \\
& +\frac{1}{6(1-2 \sigma)} U_{3 b}^{* \prime *} \zeta^{3}+\frac{1}{24(1-\sigma)} U_{3 a}^{* \prime *} \zeta^{4}-\frac{\sigma}{24(1-\sigma)} w_{2 b}^{\prime * *} \zeta^{*} \\
& \left.+\frac{2+j}{720(1-\sigma)} \omega_{0 b}^{\prime * * * *} \zeta^{6}\right] t^{6}+\cdots
\end{aligned}
$$

† We agree that $(65 c)$ sufficiently characterizes the axial case. If both $(65 c)$ and $(65 d)$ obtain, we have the pure axial case. An analogous distinction might be made in the radial vase; then (67) would refer to the pure radial case. 
If there are no surface tractions ( $S^{3} \equiv 0$ ), we suppress the terms involving $U_{3 b}$ and $w_{4 a}$ and find $U(r, 0)=0$; that is, the middle plane is bent without extension. Examples of this type are treated in $\$ \S 9,10$. In the examples of $\$ \S 11-15$ we have $S^{3} \neq 0$, and the terms in $U_{3 b}$ and $w_{4 a}$ must be retained; indeed it is precisely these terms which provide in the second of these examples $(\S 12)$ for a uniform stretching of the middle plane.

Considering only the leading terms in (69), we see that to a first approximation a linear element originally normal to $z=0$ remains straight in the strained state, is not compressed transversely, and also meets at right angles the surface into which the middle plane is bent.

In the axial case it is particularly interesting to form the physical picture for transformations $(64 a)$ and $(64 d)$, and observe how, after the plate has been turned upside down, the displacements are still correctly given by virtue of $(U,--U)$.

We observe that the $U_{0 b}$ system predominates in the radial case, with the $w_{1 b}$ system entering as an auxiliary system of axial type. The situation is reversed in the axial case; here the $w_{0 b}$ system comes first, and the $U_{1 b}$ system of radial type provides for radial displacements of higher order than the axial displacements. In the pure radial and axial cases the auxiliary $w_{1 b}$ and $U_{1 b}$ systems disappear. Of course the solutions of the radial and axial cases may be superposed if desired; that is, the most general problem may be solved by a synthesis of solutions of radial and axial type.

It may be noted that the energy integral,

$$
\begin{aligned}
& \frac{\pi E}{2(1+\sigma)(1-2 \sigma)} \int_{r_{1}}^{r_{0}} \int_{-\alpha}^{a}\left\{2(1-\sigma)\left[U^{*}+\frac{1}{t} \frac{\partial w}{\partial \zeta}\right]^{2} t\right. \\
& \left.\quad+(1-2 \sigma)\left[\left(\frac{1}{t} \frac{\partial U}{\partial \zeta}+w^{\prime}\right)^{2} t-4 \frac{U U^{\prime}}{r} t-4 U \frac{\partial w}{\partial \zeta}\right]\right\} r d r d \zeta
\end{aligned}
$$

is of order $t$ in the radial case and of order $t^{3}$ in the axial case, in agreement with the known facts.

Our formulas are in general power series in both $z$ and $h$. When $z=0$, we have power series in $h$ alone. Also the applied tractions, (18) and (55), are given by power series in $h$ alone.

Although we take the point of view of tractions applied at the faces of the plate, there is the possibility of prescribing the displacements at the faces and subsequently computing the corresponding applied tractions. So long as the prescribed elements define a determinate problem, it seems clear that the method of series is applicable. 
Finally, we note that $t$ is not necessarily a small parameter. What is important is that the actual plate under consideration be thin; that is, when the parameter $t$ is suppressed, the ratio of the thickness of the plate to its diameter must be in generalt small.

Further remarks will be found in a concluding paragraph which follows the applications.

\section{PAR'T 1I. APPLICATIONS}

7. Incomplete circular plate under internal and external radial pressure. As an example of the radial case, with the displacements given by (67), consider a plate of constant thickness with outer and inner radii $r_{0}$ and $r_{1}$ respectively. In the notation agreed upon, the thickness is $2 a=2 h$ or $2 \alpha t=2 \eta t$.

If the pressures (per unit of edge-line arc) are $p_{0}$ and $p_{1}$ on the external and internal boundaries respectively, we have

$$
\begin{aligned}
& \mathrm{T}\left(r_{0}\right)=\mathrm{T}_{1}\left(r_{0}\right) t=-\int_{-\eta}^{\eta} p_{0} t d \zeta=-2 \eta t p_{0}, \\
& \mathrm{~T}\left(r_{1}\right)=\mathrm{T}_{1}\left(r_{1}\right) t=-\int_{-\eta}^{\eta} p_{1} t d \zeta=-2 \eta t p_{1},
\end{aligned}
$$

and assuming $\mathrm{S}_{0}^{0} \equiv 0$, we have, by $(61 a)$,

$$
\begin{aligned}
& \frac{E}{1-\sigma^{2}}\left[U_{0 b}^{\prime}+\sigma \frac{U_{0 b}}{r}\right]_{r=r_{0}}=-p_{0}, \\
& \frac{E}{1-\sigma^{2}}\left[U_{0 b}^{\prime}+\sigma \frac{U_{0 b}}{r}\right]_{r=r_{1}}=-p_{1} .
\end{aligned}
$$

The first differential equation to be solved is (42). When $\alpha^{\prime}=0$, this equation reduces to

$$
U_{0 b}^{* \prime}=0
$$

† When the method leads to results which hold for a thick plate (see Part II), we have an exceptional case.

† The solution of essentially the same problem is found in Love (p. 141); in point of fact, the applications of $\$ \$ 9-12$ to plates of constant thickness do not lead to results that are new. It is enough that these examples serve admirably to illustrate the directness of the present method, as well as to suggest the detailed manner of procedure in cases that are new, notably those of variable thickness. 
and its complete solution is

$$
U_{0 b}=C_{1} r+C_{2} r^{-1}
$$

The constants are determined by (71), and have the values

$$
\begin{aligned}
& C_{1}=\frac{1-\sigma}{E}\left[\frac{p_{1} r_{1}^{2}-p_{0} r_{0}^{2}}{r_{0}^{2}-r_{1}^{2}}\right], \\
& C_{2}=\frac{1+\sigma}{E}\left[\frac{\left(p_{1}-p_{0}\right) r_{0}^{2} r_{1}^{2}}{r_{0}^{2}-r_{1}^{2}}\right] .
\end{aligned}
$$

All remaining unknowns appearing in (67) may be shown to vanish identically. For example, equations (43a) and (44) reduce to

$$
\begin{gathered}
\sigma U_{2 b}^{*}+(1-\sigma) u_{8 a}=0 \\
(1-\sigma) U_{2 b}^{* \prime}+w_{8 a}^{\prime}=0
\end{gathered}
$$

hence $U_{2 b}^{* \prime}=0$, or

$$
U_{2 b}=C_{1}^{\prime} r+C_{2}^{\prime} r^{-1}
$$

But (61c) gives

$$
\begin{aligned}
& {\left[U_{2 b}^{\prime}+\sigma \cdot \frac{U_{2 b}}{r}\right]_{r=r_{0}}=0,} \\
& {\left[U_{2 b}^{\prime}+\sigma \frac{U_{2 b}}{r}\right]_{r=r_{1}}=0,}
\end{aligned}
$$

and hence $U_{2 b} \equiv 0$. Also, $w_{8 a} \equiv 0$.

Since all unknowns vanish save $U_{0 b}$, the complete solution, as given by (67), is the following:

(75a) $U=\frac{1-\sigma}{E}\left[\frac{p_{1} r_{1}^{2}-p_{0} r_{0}^{2}}{r_{0}^{2}-r_{1}^{2}}\right] r+\frac{1+\sigma}{E}\left[\frac{\left(p_{1}-p_{0}\right) r_{0}^{2} r_{1}^{2}}{r_{0}^{2}-r_{1}^{2}}\right] \frac{1}{r}$

$$
w=-\frac{2 \sigma}{E}\left[\frac{p_{1} r_{1}^{2}-p_{0} r_{0}^{2}}{r_{0}^{2}-r_{1}^{2}}\right] z
$$


where we have made the return to the original variable $z$. From $(75 b)$, we see that there is uniform axial extension of amount given by the coefficient of $z$.

If the thickness of the plate is to remain constant, it is necessary to impose a tension. Since there is no axial extension, $w_{1 a}$ must vanish; or, by (33b),

$$
U_{0 b}^{*}=\frac{(1+\sigma)(1-2 \sigma) \mathrm{S}_{0}^{0}}{\sigma E}
$$

This relation may be used to eliminate $S_{0}^{0}$ from $(61 a)$, so that our boundary conditions become

$$
\begin{aligned}
& \frac{E}{(1+\sigma)(1-2 \sigma)}\left[(1-\sigma) U_{0 b}+\sigma \frac{U_{0 b}}{r}\right]_{r=r_{0}}=-p_{0} \\
& \frac{E}{(1+\sigma)(1-2 \sigma)}\left[(1-\sigma) U_{0 b}+\sigma \frac{U_{0 b}}{r}\right]_{r=r_{1}}=-p_{1} .
\end{aligned}
$$

Taking $U_{0 b}$ in the form

$$
U_{0 b}=K_{1} r+K_{2} r^{-1}
$$

we obtain from (77) the following values for the constants:

$$
\begin{aligned}
& K_{1}=\frac{(1+\sigma)(1-2 \sigma)}{E}\left[\frac{p_{1} r_{1}^{2}-p_{0} r_{0}^{2}}{r_{0}^{2}-r_{1}^{2}}\right], \\
& K_{\Psi}=C_{2}
\end{aligned}
$$

and we have for the complete solution in the case of no axial extension

(80a) $U=\frac{(1+\sigma)(1-2 \sigma)}{E}\left[\frac{p_{1} r_{1}^{2}-p_{0} r_{0}^{2}}{r_{0}^{2}-r_{1}^{2}}\right] r+\frac{1+\sigma}{E}\left[\frac{\left(p_{1}-p_{0}\right) r_{0}^{2} r_{1}^{2}}{r_{0}^{2}-r_{1}^{2}}\right] \frac{1}{r}$ (80b) $w=0$.

Having determined the constants in (78), we may compute $U_{0 b}^{*}$ and obtain from (76) the value of the imposed tension. We find

$$
\mathrm{S}_{0}^{0}=\frac{\sigma E}{(1+\sigma)(1-2 \sigma)} U_{0 b}^{*}=\frac{2 \sigma\left(p_{1} r_{1}-p_{0} r_{0}^{2}\right)}{r_{0}^{2}-r_{1}^{2}}
$$


In the first case considered $\left(\mathrm{S}_{0}^{0} \equiv 0\right)$, let us suppose that $p_{0}=0$; then we have $\overparen{z z} \equiv \overparen{z r} \equiv 0$, and

$$
\begin{aligned}
& \widehat{r r}=\frac{p_{1} r_{1}^{2}}{r_{0}^{2}-r_{1}^{2}}\left[1-\frac{r_{0}^{2}}{r^{2}}\right], \\
& \widehat{\theta \boldsymbol{\theta}}=\frac{p_{1} r_{1}^{2}}{r_{0}^{2}-r_{1}^{2}}\left[1+\frac{r_{0}^{2}}{r^{2}}\right] .
\end{aligned}
$$

We observe that the circumferential tension is the greatest tension; its value at $r=r_{1}$ exceeds by $p_{1}$ its value at $r=r_{0}$. The greatest extension is the circumferential extension $\left(e_{\theta \theta}\right)$ at $r=r_{1}$.

If $r_{0}-r_{1}$ is small in comparison with the thickness $2 h$, we have results that obtain for a tube rather than for a plate (cf. Love, loc. cit.).

Finally, attention may be called to the fact that we might have prescribed the radial displacements of the inner and outer edges and computed the corresponding pressures.

8. Incomplete circular plate of variable thickness under internal and external radial pressure. The displacements are given by (67), and the first equation to integrate is (42). Assuming the bases free from traction $\left(S_{0}^{0} \equiv 0\right)$, equation (42) becomes

$$
U_{0 b}^{* \prime} \alpha+\left[U_{0 b}^{\prime}+\sigma \frac{U_{0 b}}{r}\right] \alpha^{\prime}=0
$$

If we restrict $\alpha$ to be of the form

$$
\alpha(r)=\eta r^{k}\left[a(r)=h r^{k}, h=\eta t\right]
$$

equation (82) is of Euler's type and is readily transformed by a change of independent variable into a linear equation with constant coefficients. When $k=0$, we have the plate of uniform thickness already considered; but in general, the thickness at the center is either infinite or nil. We avoid this difficulty by considering an incomplete plate.

When $k=-1, h=1$, the plate is as shown below in the figure. Note that the choice of $h$ determines the thinness of the plate. We do not specialize $k$ in the discussion that follows.

† Cf. Goursat-Hedrick, Mathematical Analysis, vol. II, part II, p. 123. 


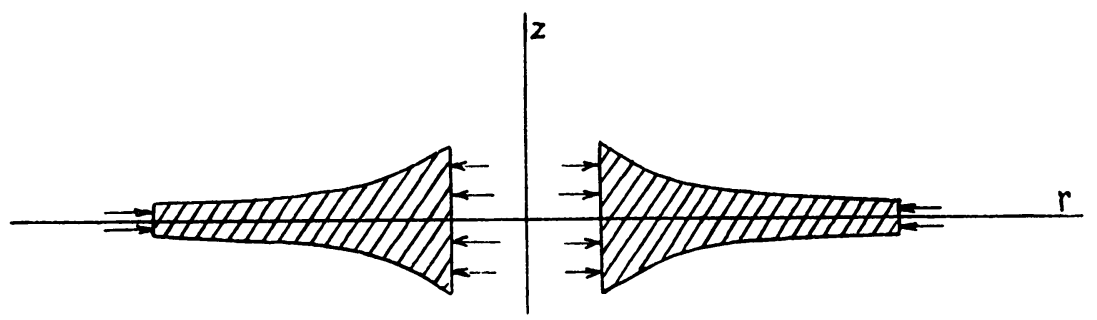

Fig. 2.

Since the ratio $\alpha^{\prime} / \alpha$ is independent of $\eta$, the leading terms in the displacements are independent of the thickness of the plate, and this is explainable when we recall the manner in which the pressures at the edges vary with the thickness.t But equations (43a) and (44) are altered by a change in $\eta$, and we see that subsequent terms in the displacements will not be independent of the thickness of the plate.

Using (83), equation (82) becomes

$$
U_{0 b}^{\prime \prime}+(1+k) \frac{U_{0 b}^{\prime}}{r}-(1-\sigma k) \frac{U_{0 b}}{r^{2}}=0,
$$

and the solution of this equation is

$$
U_{0 b}=C_{1} r^{\cdot m_{1}}+C_{2} r^{\cdot m_{2}} \text {, }
$$

where $m_{1}$ and $m_{2}$ are roots of the characteristic equation

$$
m^{2}+k m-(1-\sigma k)=0 \text {. }
$$

The discriminant of $(85 b)$ is $\Delta=k^{2}+4(1-\sigma k)$, and vanishes when $k=2 \sigma$ $\pm 2 \sqrt{\sigma^{2}-1}$; but since $\sigma<1$ (Love, p. 103), this value of $k$ is never real. Thus we see that in any bona fide physical problem, the roots of the characteristic equation are real and distinct.

If the respective pressures on the outer and inner edges are $p_{0}, p_{1}$, the conditions at the boundary are given by (71). These equations determine the constants in $(85 a)$, and we readily find

$†$ Cf. $8 \S 5,16$. 


$$
\begin{aligned}
U_{0 b}=\frac{1-\sigma^{2}}{E\left(r_{0}^{m_{1}} r_{1}^{m_{2}}-r_{0}^{m_{2}} r_{1}^{m_{1}}\right)} & {\left[\frac{r^{m_{1}}}{m_{1}+\sigma}\left(p_{1} r_{0}^{m_{2}} r_{1}-p_{0} r_{1}^{m_{2}} r_{0}\right)\right.} \\
& \left.\quad-\frac{r^{m_{2}}}{m_{2}+\sigma}\left(p_{1} r_{0}^{m_{1}} r_{1}-p_{0} r_{1}^{m_{1}} r_{0}\right)\right] .
\end{aligned}
$$

If $k=0$, then $m_{1}=1, m_{2}=-1$, and (86) reduces to the $U_{0 b}$ of the preceding paragraph.

By virtue of ( 86$)$ and $(33 b)$, we now know the leading terms in the displacements; that is,

$$
\begin{aligned}
& U=U_{0 b}+\cdots \\
& w=-\frac{\sigma}{1-\sigma} U_{0 b}^{*} z+\cdots
\end{aligned}
$$

If we wish to compute further terms in $U$ and $w$, we consider next the equations (43a) and (44). Eliminating $w_{3 a}$, we obtain the following equation for the determination of $U_{2 b}$ :

(88a) $U_{2 b}^{* \prime} \alpha+\left[U_{2 b}^{\prime}+\sigma \frac{U_{2 b}}{\gamma}\right] \alpha^{\prime}=\Phi\left(U_{0 b}, \alpha\right.$, and their derivatives $)$

Since $U_{0 b}$ and $\alpha$ are known, $\boldsymbol{\Phi}$ is a knownt function of $r$, and this equation has a solution of the form

$$
U_{2 b}=C_{1}^{\prime} r^{m}+C_{2}^{\prime} r^{m_{2}}+y^{\prime}(r)
$$

where $\varphi$ is the particular integral. By $(43 a)$, the function $w_{s a}$ is also known up to $C_{1}^{\prime}$ and $C_{2}^{\prime}$. By means of $(61 c)$, where $\mathrm{T}_{3}\left(r_{0}\right)=\mathrm{T}_{3}\left(r_{1}\right)=0$, the constants of integration are determined, and since there is no longer the homogeneity $\ddagger$ that characterized the case $k=0$, these constants are in general different from 0 . The situation here is typical for what happens as we continue to determine subsequent terms in $U$ and $w$, and it is clear that if the thickness is variable, the series for the displacements will not in general terminate.

$\dagger$ From (43), (44), (61c), we ascertain in particular the manner in which $\alpha$ and its derivatives enter in $\Phi$, and verify the fact that $U_{2 b}$ contains the factor $\eta^{2}$. Thus the term of order $t^{2}$ in $(67 a)$ is seen to be homogeneous of order 2 in $\zeta$ and $\eta$, and in general the coefficient of $t^{n}$ is homogeneous of order $n$ in $\zeta$ and $\eta$, as pointed out in $\S 1$.

$\ddagger$ Cf. $(74 d)$ and $(74 e)$. 
9. Complete circular plate loaded at its center and clamped at the edge. In this paragraph and the next three, we continue to deal with plates of constant thickness. Although we do not obtain new results, we show how the present method lends itself to direct computation of the solutions in question. Indeed, it seems desirable that in exhibiting the power of the method as applied to plates of constant thickness, we should begin with familiar rather than with new examples.

Under central load, the surface tractions are nil, and the displacements are given by (69) without the terms in $U_{3 b}$ and $w_{4 a}$. The boundary conditions are given by (59).

The first differential equation to be considered is (50), and since $\alpha^{\prime}=0$, it reduces to

$$
w_{0 b}^{\prime * * *}=0
$$

or

$$
w_{0 b}^{\prime \prime \prime}=\frac{K}{r} .
$$

The constant $K$ is determined by $(62 b)$. If $W$ is the central load, we have

$$
W=2 \pi r \mathrm{~N}_{3} t^{3},
$$

since $2 \pi r \mathrm{~N}$ is the resultant shearing force on the portion of the plate lying within a circle of radius $r$. Thus, $(89 b)$ becomes

$$
w_{0 b}^{\prime \prime \prime}=-\frac{W}{2 \pi r \delta t^{3}},
$$

and the shear is infinite at the center. Integrating, we find

$$
w_{0 b}=\frac{W}{8 \pi D}\left[r^{2} \log \frac{r_{0}}{r}+r^{2}\right]+C_{1} r^{2}+C_{2}+C_{3} \log r,
$$

where $D$ is the flexural rigidity. Since the plate is complete up to the center, $C_{3}$ must vanish. The constants $C_{1}$ and $C_{2}$ are determined by the conditions $w_{0 b}\left(r_{0}\right)=w_{0 b}^{\prime}\left(r_{0}\right)=0$, which follow from (59). We find

$$
w_{0 b}=-\frac{W}{8 \pi D}\left[\frac{1}{2}\left(r_{0}^{2}-r^{2}\right)-r^{2} \log \frac{r_{0}}{r}\right] .
$$


We turn next to the determination of $w_{2 b}$ and $U_{3 a}$. From (48), we have

$$
w_{2 b}^{\prime}+U_{3 a}=\frac{W \eta^{2}}{2 \pi r D(1-\sigma)}
$$

and equations (51) and (52) reduce to

$$
\begin{array}{r}
(95 a)-\frac{1}{2(1-\sigma)}\left[(2-\sigma) U_{3 a}^{* \prime}-\sigma w_{2 b}^{\prime * \prime}\right] \eta^{2}-\left(w_{4 b}^{\prime}+U_{5 a}\right)=0 \\
(95 b)-\frac{\sigma}{6(1-\sigma)} w_{2 b}^{\prime \prime *} \eta^{2}+\frac{2-\sigma}{6(1-\sigma)} U_{3 a}^{* \prime *} \eta^{2}-\left(w_{4 b}^{\prime}+U_{5 a}\right)^{*}=0
\end{array}
$$

Eliminating $w_{4 b}^{\prime}+U_{5 a}$ from the last two equations, and using (94) to get rid of $U_{8 a}$, the result is

$$
w_{2 b}^{\prime * *}=0
$$

or

$$
w_{2 b}^{* *}=\frac{K^{\prime}}{r}
$$

But $\mathrm{N}_{5} \equiv 0$ in $(62 f)$, and $K^{\prime}=0$; hence we find, upon integration and suppression of the term in $\log r$,

$$
w_{2 b}=C_{1}^{\prime} r^{2}+C_{2}^{\prime}
$$

Finally, the boundary conditions $w_{2 b}\left(r_{0}\right)=w_{2 b}^{\prime}\left(r_{0}\right)=0$ give us $w_{2 b} \equiv 0$, and returning to (94) we have

$$
U_{3 a}=\frac{W \eta^{2}}{2 \pi r D(1-\sigma)}
$$

The conditions $\mathrm{N}_{7} \equiv 0$ and $w_{4 b}\left(r_{0}\right)=w_{4 b}^{\prime}\left(r_{0}\right)=0$ give $w_{4 b} \equiv 0$. From (95a) the equation analogous to (94) is

$$
w_{4 b}^{\prime}+U_{5 a}=0
$$

so that the analogue to (97) is $U_{5 a} \equiv 0$. A similar argument shows that all remaining unknowns vanish identically. 
We are now in a position to write down the formulas of displacement (69). Making the return to the original variable $z$, we have

$$
\begin{aligned}
& I T=-\frac{W r z}{4 \pi D} \log \frac{r_{0}}{r}+\frac{W}{12 \pi D(1-\sigma) r}\left[6 h^{2} z-(2-\sigma) z^{3}\right] \\
& u=-\frac{W}{8 \pi D}\left[\frac{1}{2}\left(r_{0}^{2}-r^{2}\right)-r^{2} \log \frac{r_{0}}{r}\right]
\end{aligned}
$$

$$
+\frac{\sigma W z^{2}}{8 \pi D(1-\sigma)}\left[2 \log \frac{r_{0}}{r}-1\right]
$$

The central deflection is

$$
d=w(0,0)=-\frac{W r_{0}^{2}}{16 \pi D}
$$

and by means of this relation it is possible to express the displacements in terms of an initially prescribed central deflection. Indeed, it is possible to solve the problem from the point of view of an initially prescribed central deflection and subsequently compute the load in terms of $d$. We follow this suggestion in the next paragraph and obtain some interesting results.

We observe that the terms of higher order in (99) become infinite at the center.t But for a thin plate, the formulas yield reasonable displacements outside a certain small neighborhood of the axis. If $z=0$, we have the formula of the approximate theory (Love, p. 494). A meridian cut of the surface into which the middle plane is bent has infinite curvature at $r=0$.

10. Complete circular plate loaded at its center and supported at the edget. As in the preceding paragraph, we have

$$
w_{0 b}=\frac{W}{8 \pi D}\left[r^{2} \log \frac{r_{0}}{r}+r^{2}\right]+C_{1} r^{2}+C_{8}
$$

but the constants are to be determined by boundary conditions following from (58), namely

$$
u_{0 b}\left(r_{0}\right)=G_{s}\left(r_{0}\right)=0
$$

Applying these conditions, we obtain

$$
w_{0 b}=-\frac{W}{16 \pi D}\left[\frac{3+\sigma}{1+\sigma}\left(r_{0}^{2}-r^{2}\right)-2 r^{2} \log \frac{r_{0}}{r}\right]
$$

+ See the remark at the close of $\$ 10$ 
Turning to the unknowns $w_{2 b}$ and $U_{3 a}$, we find again an equation of the form $(96 c)$, and since $w_{2 b}\left(r_{0}\right)=0$, we have

$$
w_{2 b}=K\left(r^{2}-r_{0}^{2}\right)
$$

To determine $K$, we use $(63 c)$, where $\mathrm{G}_{5}\left(r_{0}\right)=0$. When $w_{2 b}$ is known, $U_{B a}$ is determined by (48), and the final results are as follows:

$$
\begin{aligned}
w_{2 b} & =-\frac{(8+\sigma) W \eta^{2}}{40(1+\sigma) \pi D r_{0}^{2}}\left(r^{2}-r_{0}^{2}\right) \\
U_{3 a} & =\frac{(8+\sigma) W \eta^{2} v}{20(1+\sigma) \pi D r_{0}^{2}}+\frac{W \eta^{2}}{2(1-\sigma) \pi D r} .
\end{aligned}
$$

From $w_{4 b}\left(r_{0}\right)=\mathrm{G}_{7}\left(r_{0}\right)=0$, we find $w_{4 b} \equiv U_{5 a} \equiv 0$, and since all further unknowns vanish identically, we may write down the following complete solution:

(106a) $\quad U=-\frac{W r}{8 \pi D}\left[2 \log \frac{r_{0}}{r}-1+\frac{3+\sigma}{1+\sigma}\right] z$

$$
+\frac{W}{4 \pi D}\left[\frac{(8+\sigma) r h^{2} z}{5(1+\sigma) r_{0}^{2}}+\frac{2 h^{2} z}{(1-\sigma) r}-\frac{(2-\sigma) z^{3}}{3(1-\sigma) r}\right]
$$

$(106 b) \quad w=-\frac{W}{16 \pi D}\left[\frac{3+\sigma}{1+\sigma}\left(r_{0}^{2}-r^{2}\right)-2 r^{2} \log \frac{r_{0}}{r}\right]$

$$
\begin{gathered}
+\frac{W}{8 \pi D}\left[\frac{(8+\sigma)\left(r_{0}^{2}-r^{2}\right) h^{2}}{5(1+\sigma) r_{0}^{2}}+\frac{\sigma}{1-\sigma}\left\{2\left(\log \frac{r_{0}}{r}-1\right)+\frac{3+\sigma}{1+\sigma}\right\} z^{2}\right] \\
-\frac{\sigma(8+\sigma) W h^{2} z^{2}}{20\left(1-\sigma^{2}\right) \pi D r_{0}^{2}} .
\end{gathered}
$$

The central deflection is

$$
d=-\frac{(3+\sigma) W r_{0}^{2}}{16(1+\sigma) \pi D}\left[1-\frac{2(8+\sigma) h^{2}}{5(3+\sigma) r_{0}^{2}}\right]
$$


Solving for $W$ in terms of $d$, we find

$(108 a)$

$$
W=-\frac{16(1+\sigma) \pi D d}{(3+\sigma) r_{0}^{2}}\left[\frac{1}{1-\frac{2(8+\sigma) h^{2}}{5(3+\sigma) r_{0}^{2}}}\right]
$$

Substituting this value in (106), we obtain formulas of displacement in terms of $d$, and these are closed forms; that is, when the parameter $t$ is introduced, these formulas of displacement are not power series in $t$. But by expanding $(108 a)$ in series,

$$
W=-\frac{16(1+\sigma) \pi D d}{(3+\sigma) r_{0}^{2}}\left[1+\frac{2(8+\sigma) h^{2}}{5(3+\sigma) r_{0}^{2}}+\cdots\right],
$$

we obtain for $U$ and $w$ the same formal developments in $t$ which result when the problem is solved from the point of view of an initially prescribed central deflection.t If we were not to write out in full these series for $U$ and $w$ in terms of the central deflection, we might fail to observe a situation that obtains in all these cases, namely, the coefficient of $t^{n}$, considered as a function of $r$ and $r_{0}$, is homogeneous $\ddagger$ of order $-n$. We have

$$
\begin{gathered}
U=\frac{2 d r}{r_{0}^{2}}\left[\frac{1+\sigma}{3+\sigma}\left(2 \log \frac{r_{0}}{r}-1\right)+1\right] z \\
+\frac{4(1+\sigma) \frac{d r}{5(3+\sigma)}[}{r_{0}^{4}}\left[\left\{\frac{8+\sigma}{3+\sigma}\left(2 \log \frac{r_{0}}{r}-1\right)-\frac{10 r_{0}^{2}}{(1-\sigma) r^{2}}\right\} h^{2} z\right. \\
\left.+\frac{5(2-\sigma) r_{0}^{2}}{3(1-\sigma) r^{2}} z^{3}\right]+\cdots
\end{gathered}
$$

$$
\begin{aligned}
& w=\frac{d}{r_{0}^{2}}\left[\left(r_{0}^{2}-r^{2}\right)-\frac{2(1+\sigma)}{3+\sigma} r^{2} \log \frac{r_{0}}{r}\right] \\
& \quad-\frac{4(1+\sigma) d}{5(3+\sigma) r_{0}^{2}}\left[\frac{(8+\sigma) r^{2} h^{2}}{(3+\sigma) r_{0}^{2}} \log \frac{r_{0}}{r}\right. \\
& \left.\quad+\frac{5 \sigma}{1-\sigma}\left\{\log \frac{r_{0}}{r}-1+\frac{3+\sigma}{8(1+\sigma)}\right\} z^{2}\right]+\cdots
\end{aligned}
$$

$\dagger$ In this case, we demand that $w_{v b}(0)=d, w_{2 b}(0)=0, w_{48}(0)=0$, etc.

$\ddagger$ Cf. $\S \S 9,11-14$ (particularly the concluding remarks of $\S 14$ ). 
The developments (109) meet the requirements both of convergence and differentiability; for the series (108b) converges when the magnitude of the ratio $\frac{h}{r_{0}}$ is suitably restricted, and is independent of $z$ and $r$.

Although (99) and (106) break down at the center of the plate, they are the exact solutions under the assumption that the surface tractions are nil in the case of central load. $t$

1. Complete circular plate under uniform pressure and clamped at the edge. In this problem, and in that of the next paragraph, the displacements are given by formulas (69) with the terms in $U_{3 b}$ and $w_{4 a}$ included.

Let the upper base $(z=h)$ be subject to the pressure $p$ per unit of surface area. Then $\mathrm{S}^{\nu} \equiv \mathrm{S}^{3} t^{3} \cdots-p, \mathrm{~S}_{\nu} \equiv 0$, and equation (50) reduces to

$$
w_{0 b}^{\prime * * *}-\frac{p}{D}
$$

or, after "anti-starring",

$$
w_{0 b}^{\prime *}=-\frac{p r}{2 D}+\frac{K}{r} .
$$

To determine the constant $K$, we have the relation $(62 b)$, and since the shear must be finite at the center, we find $K=0$ and

$$
\mathrm{N}_{3} t^{3}=\frac{1}{2} \mu r
$$

If $W$ is the total load, then $W=\pi p r_{0}^{2}$, but it is convenient to retain the $p$ in the work that follows. Since the plate is complete, we suppress the term in $\log r$ in the solution of (111) and write

$$
\omega_{0 b}=-\frac{p r^{4}}{64 D}+C_{1}^{1} r^{2}+C_{2}
$$

and when the constants of integration have been determined by means of the boundary conditions $w_{0 b}\left(r_{0}\right)=w_{0 b}^{\prime}\left(r_{0}\right)=0$, we find

$$
w_{0 b}=-\frac{p}{64 D}\left(r_{0}^{2}-r^{2}\right)^{2}
$$

For $w_{2 b}$, we find an equation of the form $(96 b)$; but $K^{\prime}=0$, since the shear would otherwise become infinite at the center. Our further boundary

$\dagger$ Cf. Saint-Venant's Annotated Clebsch, Note du $§ 45$. 
conditions on $w_{2 b}$ are the same as those of $\S 9$, and hence $w_{2 b} \equiv 0$. It follows from equation (48) that

$$
U_{8 a}=\frac{p r \eta^{2}}{2(1-\sigma) D}
$$

The remaining unknowns of the $w_{0 b}$ system vanish identically.

It remains to determine the two unknowns of the $U_{1 b}$ system. Equations (46) and (47) reduce to

$$
\sigma U_{d b}+(1-\sigma) w_{4 a}=-\frac{(1-2 \sigma) p \eta^{3}}{3(1-\sigma) D}
$$

$$
(1-\sigma) U_{8 b}^{* \prime}+\sigma w_{a a}^{\prime}=0
$$

and eliminating $w_{4 a}$ we have

$$
U_{\mathbf{B b}}^{* \prime}=\mathbf{0}
$$

Now it is clear that in addition to the condition $U\left(r_{0}, 0\right)=0$ of (59), we have also $U(0,0)=0$; but this implies that $U_{3 b}\left(r_{0}\right)=U_{B b}(0)=0$, and hence we find $U_{3 b} \equiv 0$. It follows from $(116 a)$ that

$$
w_{4 a}=-\frac{(1-2 \sigma) p \eta^{3}}{3(1-\sigma)^{2} D}
$$

The displacements becomet

$$
\begin{aligned}
& U=-\frac{p r}{16 D}\left(r_{0}^{2}-r^{2}\right) z+\frac{p r}{12(1-\sigma) D}\left[6 h^{2} z-(2-\sigma) z^{3}\right] \\
& w=-\frac{p}{64 D}\left(r_{0}^{2}-r^{2}\right)^{2}+\frac{\sigma p}{16(1-\sigma) D}\left(r_{0}^{2}-2 r^{2}\right) z^{2}
\end{aligned}
$$

$$
-\frac{p}{24(1-\sigma)^{2} D}\left[8(1-2 \sigma) h^{y} z+6 h^{2} z^{2}-\left(1-\sigma^{2}\right) z^{4}\right]
$$

† These results agree with Love, p. 490. 
We observe that the middle plane is bent without radial extension. The central deflection is

$$
d=-\frac{p r_{0}^{4}}{64 D}=-\frac{W r_{0}^{2}}{64 \pi D}
$$

and by comparison with (100) is seen to be one quarter of that which would be produced by the same total load concentrated at the center.

The normal shearing force is given by (112). For the radial force and the flexural couple, we findt

$$
\begin{gathered}
\mathrm{T} \equiv \mathrm{T}_{4} t^{4}=-\frac{\sigma p h}{1-\sigma} \\
\mathrm{G} \equiv \mathrm{G}_{3} t^{3}+\mathrm{G}_{5} t^{\overline{5}}=-\frac{p}{16}\left[(1+\sigma) r_{0}^{2}-(3+\sigma) r^{2}\right]+\frac{\left(8+\sigma+\sigma^{2}\right) p h^{2}}{20(1-\sigma)}
\end{gathered}
$$

12. Complete circular plate under uniform pressure and supported at the edge. The constants in (113) are determined by the conditions $w_{0 b}\left(r_{0}\right)=\mathrm{G}_{3}\left(r_{0}\right)=0$, and we find

$$
w_{0 b}=-\frac{p}{64 D}\left[\frac{2(3+\sigma)}{1+\sigma}\left(r_{0}^{2}-r^{2}\right) r_{0}^{2}-\left(r_{0}^{4}-r^{4}\right)\right]
$$

The determination of the unknowns $w_{2 b}$ and $U_{3 a}$ follows the lines indicated in $\S 10$. We are led to the results

$$
\begin{aligned}
& w_{2 b}=-\frac{\left(8+\sigma+\sigma^{2}\right) p \eta^{2}}{40\left(1-\sigma^{2}\right) D}\left(r_{0}^{2}-r^{2}\right), \\
& U_{8 a}=\frac{\left(2+9 \sigma-\sigma^{2}\right) p \eta^{2}}{20\left(1-\sigma^{2}\right) D} r .
\end{aligned}
$$

The remaining unknowns of the $w_{0 b}$ system vanish identically.

Turning to the unknowns $U_{3 b}$ and $w_{4 a}$, we find the first of these functious given by (117). From $U_{3 b}(0)=0$, it follows that $U_{s b}=K r$, and $K$ is determined by equation $(61 d)$, where $T_{4}\left(r_{0}\right)==0$. When $U_{3 b}$ is known, $w_{4 a}$ is given by $(116 a)$. The results are as follows:

$\dagger$ Recall that $p$ is of order $t^{3}$ and $h$ of order $t$. 


$$
\begin{aligned}
& U_{8 b}=\frac{\sigma p \eta^{3} r}{3\left(1-\sigma^{2}\right) D}, \\
& w_{4 a}=-\frac{p \eta^{3}}{3\left(1-\sigma^{2}\right) D} .
\end{aligned}
$$

The displacements take the formt

$$
U=-\frac{p r}{16 D}\left[\frac{3+\sigma}{1+\sigma} r_{0}^{2}-r^{2}\right] z
$$

$$
\begin{aligned}
& \frac{p \nu}{60\left(1-\sigma^{2}\right) D}\left[20 \sigma h^{3}+3\left(2+9 \sigma-\sigma^{2}\right) h^{2} z-5(1+\sigma)(2-\sigma) z^{3}\right], \\
& w=-\frac{p}{64 D}\left[\frac{2(3+\sigma)}{1+\sigma}\left(r_{0}^{2}-r^{2}\right) r_{0}^{2}-\left(r_{0}^{4}-r^{4}\right)\right] \\
& -\frac{p \sigma}{8(1-\sigma) D}\left[\frac{8+\sigma+\sigma^{2}}{5 \sigma(1+\sigma)}\left(r_{0}^{2}-r^{2}\right) h^{2}-\left(\frac{3+\sigma}{2(1+\sigma)} r_{0}^{2}-r^{2}\right) z^{2}\right] \\
& -\frac{p}{120\left(1-\sigma^{2}\right) D}\left[40 h^{3} z+6\left(5+2 \sigma+\sigma^{2}\right) h^{2} z^{2}-5(1+\sigma)^{2} z^{4}\right] .
\end{aligned}
$$

The shearing force is given by (112), $\mathrm{T}$ vanishes throughout the plate, and for the flexural couple we find

$$
\mathrm{G} \equiv \mathrm{G}_{3} t^{3}=-\frac{(3+\sigma) \underline{p}}{16}\left(r_{0}^{2}-r^{2}\right) \text {. }
$$

By setting $z=0$ in $(128 b)$, we obtain the expression for the surface into which the middle plane is bent. In particular, the central deflection is

$$
d=-\frac{(5+\sigma) W r_{0}^{2}}{64(1+\sigma) \pi D}\left[1+\frac{8\left(8+\sigma+\sigma^{2}\right) h^{2}}{5(1-\sigma)(5+\sigma) r_{0}^{2}}\right]
$$

where $W=\pi p r_{0}^{2}$ is the total load. The middle plane is stretched uniformly; for setting $z=0$ in $(128 a)$ we find

$$
U(r, 0)=\frac{\sigma \mu r h^{3}}{3\left(1-\sigma^{2}\right) D}=\frac{\sigma \mu r}{2 E} .
$$

† Cf. Love, p. 487. 
A linear element originally normal to $z=0$ is deformed into a curved filament; the angle at which this curve cuts the surface into which the middle plane is bent is

$$
\theta=\frac{1}{2} \pi-\lambda,
$$

where $\lambda$ is given with sufficient accuracy by the tangent

$$
\tan \lambda=\left.\frac{\frac{\partial U}{\partial z}+w^{\prime}}{1--\frac{\partial U}{\partial z} w^{\prime}}\right|_{z:=0}
$$

This formulation leads to the result

$$
H=\frac{1}{2} \pi-\frac{3(1+\sigma) \underline{p r}}{4 E h} .
$$

When we solve the example of this paragraph (or the preceding) from the point of view of an initially prescribed central deflection, $t$ we are led to a situation analogous to that noted in $\S 10$.

13. Plates of constant thickness under pressure of type such that the series for the displacements terminate. The "star" notation, besides rendering our formulas compact, enables us to see with ease the types of loading that lead to terminating series for the displacements.

Let us look first at the axial case. In the coefficient of $t^{2 n}$ in $(69 b)$, we observe that $w_{0 b}$ is subject $n$ times to the pair of operations ${ }^{\prime *}$; for example,

† To solve this problem $a b$ initio means that we must retain the odd S's which enter in the differential equations of the $U_{1 b}$ and $w_{0 b}$ systems. Since we seek a uniform loading that will cause a central deflection $;$, we find $w_{v b}^{\prime * * *}=$ const., $w_{2 b}^{\prime * \prime *}=$ const., etc. Integrating and suppressing the log term, there are three constants to be determined. For the first equation, the boundary conditions are $w_{0 b}(0)=d, w_{08}\left(r_{0}\right)=G_{8}\left(r_{0}\right)=0$; subsequent boundary conditions are of the type $v_{2 b}(0)=w_{2 b}\left(r_{0}\right)=G_{b}\left(r_{0}\right)=0$, without the $d$. We are thus led to the result

$$
\begin{aligned}
-p & =\mathrm{S}^{3} t^{3}+\mathrm{S}^{5} t^{j}+\cdots=D w_{0 b}^{\prime * *}+D w_{2 b}^{\prime * \prime *} t^{2}+\cdots \\
& =\frac{64(1+\sigma) D d}{(5+\sigma) r_{0}^{4}}\left[1-\frac{8\left(8+\sigma+\sigma^{2}\right) h^{2}}{5(1-\sigma)(5+\sigma) r_{0}^{2}}+\cdots\right]
\end{aligned}
$$

which is precisely what we get by solving (130a) for $p$ in terms of $d$ and expanding. 
the term in $t^{6}$ contains $w_{0 b}^{\prime * * * *}$. A similar situation presents itself in $(69 a)$, except that here the final operation in the sequence is that of differentiation. Equation (50) reduces for the case of constant thickness to

$$
w_{0 b}^{\prime \prime * *}=\frac{s^{3} t^{3}}{D}
$$

and it appears that the series for the displacements will terminate if and only if the pair of operations ${ }^{* *}$, applied a sufficiert number of times to (134), causes the right-hand member of the equation to vanish. The truth of this conjecture is borne out by the structure of the differential equations of the $w_{0 b}$ system. We are assuming, of course, that the load is prescribed at the outset.

A little consideration shows that the only type of loading that meets the stated requirement has the form

$$
\sum_{n=0}^{m}-\mu_{2 n} r^{2 n}+\log r \sum_{n=0}^{m}-q_{2 n} r^{2 n}
$$

Here the $p$ 's and $q$ 's are constants, positive in the case of pressure. We confine our attention to pressures of the form

$$
\sum_{n=0}^{m}-p_{2 n} r^{2 n}
$$

for terms in the logarithm give a type of loading which not only lacks physical interest but also leads to formulas of displacement which present discontinuities at the center of the plate.

Although a load of the form

$$
\sum_{n=0}^{m}-p_{2 n+1} r^{2 n+1}
$$

fails to lead to terminating series for the displacements, it presents some features of interest. With this type of pressure, the successive processes of differentiation and starring lead at a certain stage to the starring of a constant or a constant multiplied by $\log r$. At this point, negative powers of $r$ are introduced, and since the next operation is that of differentiation it is clear that no term can reduce to 0 at any stage. In this type of loading not only are the displacements given by infinite series, but also, due to the entrance of negative powers of $r$, all terms from a certain point on will present discontinuities at the center of the plate.

The phenomenon just pointed out is not without explanation. Turning to $(135 b)$ and (136), we are led to examine the curve $z=r^{n}$ with reference to 
its behavior at the origin. If $n$ is even, as in $(135 b)$, the curve is continuous at $r=0$, together with all its derivatives. When $n$ is odd, as in (136), the $n$th derivative is discontinuous at $r=0$ and has there a jump of amount $2 n$ !, and it is natural that such a singularity should manifest itself in the formulas of displacement.

It will be recalled that we assumed $S_{0}^{0}$ constant; in general it is a function of $r$, and if the series for the displacements are to terminate in the radial case, $\mathrm{S}_{0}^{0}$ must clearly have form (135). Otherwise the successive differentiation and starring operations would at no stage lead to 0 .

When $n=0,(135 b)$ yields the important case of uniform loading - and if $p_{0}=0$, central loading. The latter case is dealt with fully in $\$ \S 9,10$. In the case of uniform loading, and for $(135 b)$ in general, a number of interesting possibilities arise when the plate is taken to be incomplete. In point of fact, we may have

(a) both edges clamped,

(b) both edges supported,

(c) one edge clamped and one edge free,

(d) one edge clamped and one edge supported,

(e) one edge supported and one edge free.

In cases $(c),(d),(e)$ we may interchange the rôle of inner and outer edges. Furthermore, we obtain results for a complete plate by setting the inner radius equal to 0 in any of these three cases. We are not attempting to indicate in any exhaustive manner the full scope of the applications, but it is of some interest to note the variety of problems that lead to terminating series for the displacements.

In the following paragraph, we consider an incomplete plate, mainly because we wish to paraphrase the problem (in $\S 15$ ) for the case of variable thickness, but in this example we take the pressure to be uniform in order to simplify the computations. It may be well to have on record an example in which the load depends upon $r$. It is not a long piece of work to compute the following displacements for the case of a complete plate, clamped at $r=r_{0}$ and supporting a load $-p r^{2}$ :

$$
\text { (137a) } \quad \begin{aligned}
& U=-\frac{p r}{96 D}\left(r_{0}^{4}-r^{4}\right) z-\frac{p r}{4(1-\sigma) D}\left[\frac{\sigma\left(r_{0}^{2}-r^{2}\right) h^{3}}{3}\right] \\
& -\left\{r^{2}+\frac{(8-3 \sigma)\left(r_{0}^{2}-r^{2}\right)}{10}\right\} h^{z} z+\frac{(2-\sigma) r^{2} z^{3}}{6(1-\sigma)} \\
& +\frac{p r}{60(1-\sigma) D}\left[4 h^{4} z+20(1-\sigma) h^{3} z^{2}+40(1-3 \sigma) h^{2} z^{3}+(3-\sigma) z^{5}\right],
\end{aligned}
$$




$$
\begin{aligned}
u & =-\frac{p}{576 D}\left[2 r_{0}^{6}-3 r_{0}^{4} r^{4}+r^{6}\right] \\
& +\frac{p}{480(1-\sigma) D}\left[3(8-3 \sigma)\left(r_{0}^{2}-r^{2}\right)^{2} h^{2}+5 \sigma\left(r_{0}^{4}-3 r^{4}\right) z^{2}\right] \\
& +\frac{p}{120(1-\sigma)^{2} D}\left[20\left\{\sigma^{2} r_{0}^{2}-2(1-\sigma)^{2} r^{2}\right\} h^{3} z\right. \\
& \left.-3\left\{10 r^{2}+\sigma(8-3 \sigma)\left(r_{0}^{2}-2 r^{2}\right)\right\} h^{2} z^{2}+5\left(1-\sigma^{2}\right) r^{2} z^{4}\right] \\
& -\frac{p}{180(1-\sigma)^{2} D}\left[6 h^{4} z^{2}+40 \sigma(1-\sigma) h^{3} z^{3}-3(1-\sigma)(2-3 \sigma) h^{2} z^{4}\right. \\
& \left.+(1-\sigma)(2+\sigma) z^{6}\right] .
\end{aligned}
$$

The central deflection is

$$
d=-\frac{p r_{0}^{6}}{288 D}+\frac{(8-3 \sigma) p h^{2} r_{0}^{4}}{160(1-\sigma) D}
$$

When $U$ and $w$ are expressed in terms of $d$, the coefficients, considered as functions of $r$ and $r_{0}$, are homogeneous and of order in agreement with the rule given at the close of $\S 10$. If $U$ and $w$ are written in terms of $p$. or in terms of the total load $\left(W=\frac{1}{4} \pi p r_{0}^{4}\right)$, there is still homogeneity in $r$ and $r_{0}$, but in such cases the order is altered throughout by one and the same constant.t

14. Incomplete circular plate under uniform pressure with one edge clamped and one edge free. Taking $r=r_{1}$ as the free edge, we determine the constant in equation (111) by means of the condition $\mathrm{N}_{3}\left(r_{1}\right)=0$ and find

$$
\prime \prime^{\prime \prime \prime b}=-\frac{p}{2 D r}\left(r^{2}-r_{1}^{2}\right)
$$

It follows that

$$
\mathrm{N}_{3}\left(r_{0}\right) t^{3}=\frac{p}{2 r_{0}}\left(r_{0}^{2}-r_{1}^{2}\right)
$$

We assume that $r_{1}$ is the inner edge; if $r_{1}>r_{0}$, the parenthesis changes sigu. in agreement with the conventions of $\S 5$.

$\dagger$ See the concluding remarks of $\$ 14$. 
Integrating (139), the three constants are determined by the conditions $G_{8}\left(r_{1}\right)=w_{0 b}\left(r_{0}\right)=w_{0 b}^{\prime}\left(r_{0}\right)=0$, and the final result is the following:

$$
\begin{aligned}
w_{0 b} & =-\frac{p}{32 Q D}\left[\left\{(1-\sigma)\left(r_{0}^{2}+2 r_{1}^{2}\right) r_{0}^{2}+(1+3 \sigma) r_{1}^{4}\right\}\left(r_{0}^{2}-r^{2}\right)\right. \\
& +2 r_{0}^{2} r_{1}^{2}\left\{(1+\sigma) r_{0}^{2}+(1-\sigma)\left(2 r^{2}+r_{1}^{2}\right)\right\} \log \frac{r_{0}}{r} \\
& \left.+4(1+\sigma) r_{1}^{4}\left\{r_{0}^{2} \log \frac{r_{0}}{r_{1}}-r^{2} \log \frac{r}{r_{1}}\right\}-8(1+\sigma) r_{0}^{2} r_{1}^{4} \log \frac{r_{0}}{r} \log \frac{r_{0}}{r_{1}}\right] \\
& +\frac{p}{64 D}\left[r_{0}^{4}-r^{4}+8\left(r_{0}^{2}-r^{2}\right) r_{1}^{2}\right],
\end{aligned}
$$

where the constant $Q$ is given by

$$
Q=(1-\sigma) r_{0}^{2}+(1+\sigma) r_{1}^{2}
$$

When we set $r_{1}=0,(141)$ reduces to (114).

For $w_{2 b}$, we have an equation of the form $(96 b)$, where the condition $\mathrm{N}_{5}\left(r_{1}\right)=0$ shows the constant must be 0 . Hence we have to integrate the equation

$$
w_{2 b}^{\prime \prime \prime}=0
$$

and determine the three constants by means of the conditions $\mathrm{G}_{b}\left(r_{1}\right)=w_{2 b}\left(r_{0}\right)=w_{2 b}^{\prime}\left(r_{0}\right)=0$. Having found $w_{2 b}$, equation (48) gives us $U_{8 a}$. The results are as follows:

$$
\begin{aligned}
& W_{2 b}=-\frac{(8-3 \sigma) p r_{1}^{2} \eta^{2}}{20(1-\sigma) Q D}\left[r_{0}^{2}-r^{2}-2 r_{0}^{2} \log \frac{r_{0}}{r}\right] \\
& U_{8 a}=\frac{\left(r^{2}-r_{1}^{2}\right) p \eta^{2}}{2(1-\sigma) D r}+\frac{(8-3 \sigma)\left(r_{0}^{2}-r^{2}\right) p r_{1}^{2} \eta^{2}}{10(1-\sigma) Q D \vartheta} .
\end{aligned}
$$

For $U_{8 b}$, we have equation (117). The conditions $U_{8 b}\left(r_{0}\right)=\mathrm{T}_{4}\left(r_{1}\right)=0$ give $U_{8 b} \equiv 0$. It follows that $w_{4 a}$ is given by (118). 
Substitution in (69) gives the following displacements :

(144a)

$$
\begin{aligned}
U & =-\frac{p r}{16 Q D}\left[(1-\sigma)\left(r_{0}^{2}+2 r_{1}^{2}\right) r_{0}^{2}+(1+3 \sigma) r_{1}^{4}\right. \\
& +\left\{(1+\sigma) r_{0}^{2}+(1-\sigma)\left(2 r^{2}+r_{1}^{2}\right)\right\} \frac{r_{0}^{2} r_{1}^{2}}{r^{2}}-\left(r^{2}+4 r_{1}^{2}\right) Q \\
& -\frac{4(1+\sigma) r_{0}^{2} r_{1}^{4}}{r^{2}} \log \frac{r_{0}}{r_{1}}+2(1+\sigma) r_{1}^{4}\left\{2 \log \frac{r}{r_{1}}+1\right\}
\end{aligned}
$$

$$
\begin{aligned}
& \left.-4(1-\sigma) r_{0}^{2} r_{1}^{2} \log \frac{r_{0}}{r}\right] z \\
& +\frac{p}{12(1-\sigma) D r}\left[6\left\{r^{2}-r_{1}^{2}+\frac{(8-3 \sigma)\left(r_{0}^{2}-r^{2}\right) r_{1}^{2}}{5 Q}\right\} h^{2} z\right. \\
& \left.-(2-\sigma)\left(r^{2}-r_{1}^{2}\right) z^{8}\right],
\end{aligned}
$$$$
w=w_{0 b} \text { (as given above) }
$$

$$
\begin{aligned}
& -\frac{p}{80(1-\sigma) Q D}\left[4(8-3 \sigma) r_{1}^{2}\left\{r_{0}^{2}-r^{2}-2 r_{0}^{2} \log \frac{r_{0}}{r}\right\} h^{2}\right. \\
& -5 \sigma\left\{4(1+\sigma) r_{1}^{4}\left(\log \frac{r}{r_{1}}+1\right)-4(1-\sigma) r_{0}^{2} r_{1}^{2}\left(\log \frac{r_{0}}{r}-1\right)\right.
\end{aligned}
$$

(144b)

$$
\begin{aligned}
& \left.\left.+(1-\sigma)\left(r_{0}^{2}+2 r_{1}^{2}\right) r_{0}^{2}+(1+3 \sigma) r_{1}^{4}-\left(2 r^{2}+4 r_{1}^{2}\right) Q\right\} z^{2}\right] \\
& -\frac{p}{120(1-\sigma)^{2} D}\left[40(1-2 \sigma) h^{3} z+30 h^{2} z^{2}\right. \\
& \left.-\frac{12 \sigma(8-3 \sigma) r_{1}^{2} h^{2} z^{2}}{Q}-5\left(1-\sigma^{2}\right) z^{4}\right]
\end{aligned}
$$

When $r_{1}=0$, these formulas reduce to (119). If we think of $r_{0}$ as the inner edge, we see that by setting $r_{0}=0$ we obtain the solution for the case of a complete plate under uniform pressure with center clamped and edge free.

In the examples of complete plates, $\$ \S 9-13$, we found the coefficients in $U$ and $w$ homogeneous in $r$ and $r_{0}$; when the plate is incomplete, there is homogeneity in $r, r_{0}$ and $r_{1}$. For example, we observe in (144), where $U$ and $r$ : 
are expressed in terms of $p$, that the coefficient of $t^{n}$ is homogeneous of order $4-n$ in $r, r_{0}$ and $r_{1}$.

The "check of dimensions" furnishes the clue to the situation in all cases. For example, when $U$ and $w$ are expressed in terms of $d$, all coefficients of $d$. must clearly be of the 0th degree in length. We are thus led to the result that the coefficient of $t^{n}$ must be of the 0th degree in length. But being homogeneous of order $n$ in $\zeta$ and $\eta$, this coefficient is of the $n$th degree in length so far as $z$ and $h$ are concerned. It follows that the contributions made by $r, r_{0}$ and $r_{1}$ must total to degree $-n$.

The check of dimensions does not require that we write the displacements in terms of $d$, or that the plate be complete, or even of constant thickness. It is important to note that we have finally obtained a maximum amount of information as to the manner of occurrence not only of $\zeta$ and $\eta$, and $\alpha$ and its derivatives, but also of $r, r_{0}$ and $r_{1}$. It is a particular advantage to have this information available as an aid to the elimination of errors in computation.

15. Incomplete circular plate of variable thickness under uniform pressure with one edge clamped and one edge free. In $\$ 8$, we considered an example of variable thickness under the radial case; it remains to consider an example of variable thickness under the axial case.

Let us take $\mathrm{S}^{\nu} \equiv \mathrm{S}^{3} t^{3}=-p, \mathrm{~S}_{\nu} \equiv 0$, where $p$ is a constant pressure per unit of surface area and is everywhere normal to the upper base $z=a$. Under this assumption it is easy to anti-star equation (50); we find

$$
w_{0 b}^{\prime \prime \prime} \alpha^{3}+3\left[w_{0 b}^{\prime \prime}+\sigma \frac{w_{0}^{\prime}}{r}\right] \alpha^{2} \alpha^{\prime}-\frac{3\left(1-\sigma^{2}\right) p r}{4 E t^{3}}+\frac{K}{r} .
$$

To determine $K$, we use (62a) where $\mathrm{N}_{3}\left(r_{1}\right)=0$. When this is done, we may write our equation in the form

$$
w_{0 b}^{\prime \prime \prime}+w_{0 b}\left[\frac{1}{r}+\frac{3 a^{\prime}}{a}\right]+w_{0 b}^{\prime}\left[\frac{3 \sigma a^{\prime}}{r a}-\frac{1}{r^{2}}\right]=-\frac{3\left(1-\sigma^{2}\right) p\left(r^{2}-r_{1}^{2}\right)}{4 E a^{3} r}
$$

where $a=\alpha t$.

This equation resembles (82) in the respect that when

$$
\frac{a^{\prime}}{a}=\frac{k}{r}
$$

it is of Euler's type and readily integrated. We accordingly confine ourselves to the case that

$$
a(r)=7 r^{k}
$$


and avoid difficulty at the center by taking the plate to be incomplete. Since $k$ and $h$ are at our disposal, (148) yields a variety of interesting shapes for the plate in question.

Substituting (148) in (146), and multiplying through by $r^{3}$, the equation to be integrated takes the form

$$
w_{0 b}^{\prime \prime \prime} r^{3}+w_{0 b}^{\prime \prime} r^{2}(1+3 k)+w_{0 b}^{\prime} r(3 \sigma k-1)=-\frac{3\left(1-\sigma^{2}\right) p\left(r^{2}-r_{1}^{2}\right)}{4 E h^{3} r^{3 k-2}} .
$$

(Applying a check of dimensions, we observe that both $p$ and $h^{3}$ are of order $t^{3}$, and the right-hand member is of order $t^{0}$ as it should be.) This equation is the analogue of (139).

To solve (149) we set $v=\log r$. This change of independent variable gives us an equation with constant coefficients, and the homogeneous equation associated therewith has the characteristic equation

$$
m\left[m^{2}+(3 k-2) m-3 k(1-\sigma)\right]=0 .
$$

Here the quadratic factor has the discriminant $\Delta=(3 k-2)^{2}+12 k(1-\sigma)$, which vanishes when $k=\frac{2}{8}\left(\sigma \pm \sqrt{\sigma^{2}-1}\right)$. But since $\sigma<1$, this value of $k$ is never real. Thus we see that in any physical problem $\Delta$ will be positive, and equation (150) will have in general three distinctt real roots; we denote these by

$$
0, m_{1}, m_{2} \text {. }
$$

When we add to the solution of the homogeneous equation the particular integralł of the non-homogeneous equation, we find the complete solution of (149) to be the following:

$\dagger$ The only exception is when $k=0$.

$\ddagger$ The particular integral is found by the well known methods of Murray, Introductory Course in Differential Equations, $\$ 70$, p. 89. We exclude from the text the exceptional values $k=0, k=\frac{2}{3}, k=\frac{4}{3}, k=\frac{8}{3(3-\sigma)}$. The case $k=0$ is disposed of in $\S 14$; by the methods of Murray, p. 90, the remaining special values of $k$ lead respectively to the following particular integrals:

$$
\begin{aligned}
& k=\frac{2}{3}:-\frac{3 p}{16 E h^{3}}\left[(1-\sigma) r^{2}+2(1+\sigma) r_{1}^{2} \log r\right] ; \\
& k=\frac{4}{3}: \frac{3(1+\sigma) p}{32 E h^{3}}\left[2 \log r+\frac{r_{1}^{2}}{r^{2}}\right] \\
& k=\frac{8}{3(3-\sigma)}:-\frac{3(1+\sigma)(3-\sigma)^{2} p}{32 E h^{3}}\left[\frac{r^{4(1-\sigma) /(3-\sigma)} \log r}{\sigma-3 \sigma}-\frac{r_{1}^{2} r^{-2(1+\sigma) /(8-\sigma)}}{2(1+\sigma)}\right] .
\end{aligned}
$$




$$
w_{0 b}=K_{1} r^{m_{1}}+K_{2} r^{m_{3}}+K_{3}
$$

$-\frac{3\left(1-\sigma^{2}\right) p}{4 E h^{3}}\left[\frac{r^{4-8 k}}{2(4-3 k)^{2}-3 k(1-\sigma)(4-3 k)}+\frac{r_{1}^{2} r^{2-8 k}}{3 k(1-\sigma)(2-3 k)}\right]$.

The three constants are determined by means of the boundary conditions $\mathrm{G}_{\mathrm{B}}\left(r_{1}\right)=w_{0 b}\left(r_{0}\right)=w_{0 b}^{\prime}\left(r_{0}\right)=0$. The result is

$$
\begin{gathered}
w_{0 b}=-K_{1}\left(r_{0}^{m_{1}}-r^{m_{1}}\right)-K_{2}\left(r_{0}^{m_{1}}-r^{m_{2}}\right) \\
-\frac{3\left(1-\sigma^{2}\right) p}{4 E h^{8}}\left[\frac{r^{4-8 k}-r_{0}^{4-8 k}}{2(4-3 k)^{2}-3 k(1-\sigma)(4-3 k)}+\frac{r_{1}^{2}\left\{r^{2-8 k}-r_{0}^{2-8 k}\right\}}{3 k(1-\sigma)(2-3 k)}\right]
\end{gathered}
$$

where

$$
\begin{aligned}
& K_{1}=\frac{3\left(1-\sigma^{2}\right) p}{4 E h^{3} m_{1}}\left[\frac{A}{Q}\left(m_{2}-1+\sigma\right) r_{1}^{m_{3}}+\frac{B}{Q} r_{0}^{m_{2}}\right] \\
& K_{2}=-\frac{3\left(1-\sigma^{2}\right) p}{4 E h^{3} m_{2}}\left[\frac{A}{Q}\left(m_{1}-1+\sigma\right) r_{1}^{m_{1}}+\frac{B}{Q} r_{0}^{m_{1}}\right]
\end{aligned}
$$

and the auxiliary constants are given by

$$
\begin{aligned}
& A=\left[\frac{r_{0}^{2}}{2(4-3 k)-3 k(1-\sigma)}+\frac{r_{1}^{2}}{3 k(1-\sigma)}\right] r_{0}^{2-8 k}, \\
& B=\left[\frac{3 k-3-\sigma}{2(4-3 k)-3 k(1-\sigma)}+\frac{3 k-1-\sigma}{3 k(1-\sigma)}\right] r_{1}^{4-8 k}, \\
& Q=\left(m_{2}-1+\sigma\right) r_{0}^{m_{1}} r_{1}^{m_{1}}-\left(m_{1}-1+\sigma\right) r_{0}^{m_{2}} r_{1}^{m_{1}} .
\end{aligned}
$$

We have found the leading term in the axial displacement, and we readily compute the leading term in the radial displacement. As in $\S 8$, we find, when we proceed to the determination of further unknowns, that the differential equations are of the same type as the one just considered and hence readily integrable. The only difficulties involved are apparently in the nature of disagreeable computation.

16. Critique of method. In the case of plates of constant thickness, we have seen that the method of series leaves nothing to be desired with reference to rigor; in the examples of physical interest, the series for the 
displacements terminate, and when the parameter $t$ is suppressed, the formulas satisfy the equations of equilibrium identically. Furthermore, we have observed that when the central deflection is prescribed, the displacements are given by infinite series which meet the requirements both of convergence and differentiability.

When the thickness is variable, the formal series in $t$ for the displacements become complicated, and as yet the author has not found opportunity to examine in any detail the convergence of these more involved developments. In the nature of the method, if we suppress the parameter $t$ in the series orderea according to degree of homogeneity in $z$ and $h$, these series are such that, when differentiated formally and substituted in the equations of equilibrium, terms of like degree annul each other; that is, the equations of equilibrium are satisfied in the usual formal sense.

Furthermore, there is a sense in which the formal series for the displacements yield approximate solutions. If we break off the series and discard terms of order higher than $t^{2 k+1}$, we obtain formulas of displacement which correspond to a total surplus body force per unit of volume of order $t^{2 k}$ and a total surplus surface traction per unit of area of order $t^{2 k+1}$. Thus the truncated series correspond to a total surplus applied force of order $t^{2 k}$, whereas the total given applied force is at most of order $t^{3}$, that is, of the order of the cube of the ratio of the thickness of the plate to its diameter. If $k$ is large and the ratio of thickness to diameter is small, it seems probable that when the surplus applied forces are removed, no sensible change occurs in the displacements, and that we have a physical solution of our problem. It would be of interest to check this conjecture by comparison of the potential energy in the actual and approximate solution.

We note that the present paper is concerned with the restricted theory of strain; + that is, we have suppressed the squares and products of the derivatives of $U$ and $w$ in writing the expressions (16) for the components of strain. Recall also that our results hold only for plates slightly bent, and that we have assumed a homogeneous and isotropic material.

Our assumption of developability in the "neighborhood of the middle plane" does not permit the satisfaction of the most general type of boundary condition at a surface $r=$ const. But if the applied tractions are distributed in the manner we have specified, our solutions are exact; "if they are distributed otherwise (Love, p. 480), without ceasing to be equivalent to resultants of the types T, N, G, the solution represents the state of the plate with sufficient approximation at all points which are not close to the edge".

In the usual theory, exact solutions of the more involved problems in

† Cf. Love, p. 57. on the general theory of strain. 
circular plates (of constant thickness) are arrived at by arbitrary synthesis, through superposition, of several solutions of simpler type, the latter being obtained by methods which are not without complication. It appears that the method of series, when applied to plates of constant thickness, involves a minimum amount of computation and thus commends itself for the simplicity and directness of its underlying motif.

The method of series is applicable to a variety of one- and two-dimensional problems, + with thickness either uniform or variable, and its usefulness may extend to hydrodynamics, electricity, and electro-magnetism. In the problem of the circular plate we have been led to total differential equations. In general, there are partial differential equations to be dealt with, but this increase in complexity lies in the nature of the problem itself.

The method affords a solution of a problem which has interested elasticians since Poisson and Cauchy.

HARTARD UNIVERSITY,

CAMBRIDGE, MaSS.

January, 1923.

† The author has prepared a paper on Rods of constant or variable circular cross section. The method of series is immediately adaptable, and applications are made to the uniformly tapering rod, to a bulging rod, and to the right circular cylinder. This paper will appear in the American Journal of Mathematics.

As the page proof of the present paper leaves my hands I am applying the method of series to rectangular plates. In a number of cases of constant thickness the partial differential equations are tractable, and it is possible to obtain exact solutions; in such cases the problem of the thick plate or beam is completely solved. If we set $z=0$ in the exact solution, there remain: i) the leading term, independent of $z$ and $h$ (the solution of the approximate theory), and ii) certain terms, involving $h$, not accounted for in the approximate theory. So far as I am aware, the only contribution to the problem of the thick rectangular plate is M. Mesnager's solution for the case of supported edges and central or uniform pressure (Comptes Rendus, vol. 164, 1917, p. 721 and vol. 165, 1917, p. 551). 